\title{
A meta-analysis of effects of selective serotonin reuptake inhibitors on blood pressure in depression treatment: outcomes from placebo and serotonin and noradrenaline reuptake inhibitor controlled trials
}

This article was published in the following Dove Press journal:

Neuropsychiatric Disease and Treatment

7 November 2017

Number of times this article has been viewed

\author{
Zhuoyuan Zhongl,* \\ Limin Wang ${ }^{2, *}$ \\ Xiaojun Wen ${ }^{3}$ \\ Yunyun Liu ${ }^{4}$ \\ Yafei Fan ${ }^{5}$ \\ Zhonglin Liu $^{5}$ \\ 'Department of Neurology, The \\ People's Hospital of Guangxi \\ Zhuang Autonomous Region, \\ Nanning, China; ${ }^{2}$ Department of \\ Neurology, Guangdong General \\ Hospital, Guangdong Academy \\ of Medical Sciences, Guangdong \\ Neuroscience Institute, Guangzhou, \\ China; ${ }^{3}$ Department of Neurology, \\ Guangzhou First Municipal \\ People's Hospital, Guangzhou \\ Medical University, Guangzhou- \\ Birmingham University Brain and \\ Cognition Center, Guangzhou, \\ China; ${ }^{4}$ Department of Neurology, \\ the Sixth Affiliated Hospital Sun \\ Yat-sen University, Guangzhou, \\ China; ${ }^{5}$ Department of Neurology, \\ Sun Yat-sen Memorial Hospital, Sun \\ Yat-sen University, Guangzhou, China \\ *These authors contributed equally \\ to this work
}

Correspondence: Zhonglin Liu Department of Neurology, Sun Yat-sen Memorial Hospital of Sun Yat-sen University, 107 Yan Jiang West Road, Guangzhou, Guangdong, China

$\mathrm{Tel}+8602081332621$

Fax +86 02081332833

Email 13719438236@I26.com
Background: Selective serotonin reuptake inhibitors (SSRIs) and serotonin and noradrenaline reuptake inhibitors (SNRIs) have been commonly prescribed for depression treatment. However, their effects on blood pressure are unclear.

Materials and methods: Effects on blood pressure of depressive patients in two groups (SSRIs versus placebo and SSRIs versus SNRIs) were evaluated. A search was conducted for doubleblind, randomized controlled trials (RCTs) in PubMed, EMBASE, ISI Web of Science, PsycNET, CCRCT, and DARE (up to March 2017). The outcomes were systolic blood pressure (SBP) changes and diastolic blood pressure (DBP) changes from baseline to endpoint or to a certain period of treatment duration. Weighted mean differences (WMDs) and 95\% CIs were calculated and pooled using random effects models. The $\chi^{2}$ test and $I^{2}$ statistics were used to assess heterogeneity. Funnel plots, Begg's test, and Egger's test were used to estimate publication bias.

Results: A total of 23 RCTs involving 13,285 participants were included. Patients on SSRIs showed no significant differences in blood pressure changes compared with placebo. In the group of SSRIs versus SNRIs, overall SBP changes and DBP changes revealed statistical significances (WMD $1.5 \mathrm{mmHg}, 95 \% \mathrm{CI}-2.15,-0.84, Z=4.46, P<0.00001$ and WMD $1.34 \mathrm{mmHg}$, $95 \% \mathrm{CI}-1.92,-0.75, Z=6.18, P<0.00001)$. Subgroup analyses on treatment duration and age further evidenced these findings.

Conclusion: It was established that SSRIs did not affect blood pressure, while SNRIs led to a modest increase in SBP and DBP with statistical significance compared with SSRIs.

Keywords: meta-analysis, blood pressure change, depression treatment, selective serotonin reuptake inhibitors, serotonin and noradrenaline reuptake inhibitors, depression, antidepressant, systolic blood pressure, diastolic blood pressure

\section{Introduction}

Major depression manifests kinds of cognitive and biological symptoms, leading to a debilitating condition. Worldwide, $17 \%$ of people are likely to experience major depression during their lifetime; it is especially prevalent in patients with cardiovascular disorders. ${ }^{1}$ It is well established that depressive patients tend to have unhealthy lifestyles including smoking, physical inactivity, and poor medication adherence. This in turn exacerbates cardiovascular conditions including hypertension and left ventricular hypertrophy. ${ }^{2}$ Depressed individuals, whether with a clinical diagnosis 
of depression, have been reported to have a higher occurrence of morbidity and mortality for cardiovascular events. ${ }^{3}$ Some studies indicate that low blood pressure (BP) is associated with increased prevalence of depression, as well as with depressive symptom severity, which is independent of age, sex, or cardiovascular disease, ${ }^{4}$ even independent of baseline BP or other risk factors usually associated with hypertension. This association is not explained by the use of antidepressants or antihypertensive medications. ${ }^{5}$ The Three City Study confirms that both depressed men and women have lower systolic blood pressure (SBP) and diastolic blood pressure (DBP), which are unrelated to antihypertensive or psychotropic agents. ${ }^{6}$ In contrast, a meta-analysis suggests anxiety and hypertension are significantly correlated, drawing the conclusion that anxiety is an independent risk factor for incident hypertension. ${ }^{7}$ Anxiety and depression are closely linked. ${ }^{8}$ A previous review also showed an increased risk of hypertension in depressed patients and an increased risk of depression in hypertensive patients. ${ }^{9}$

Besides controversies regarding the connection between depression and BP level, the role of antidepressants in mood disorders such as anxiety and depression and BP is equivocal. Tricyclic antidepressants have been reported to result in higher mean SBP and DBP, thus making hypertension stage 1 more likely. ${ }^{10}$ Selective serotonin reuptake inhibitors (SSRIs) and serotonin and noradrenaline reuptake inhibitors (SNRIs), the second-generation antidepressants, are prescribed for both anxiety and depression, consisting of a tower of strength in treatment of affective disorders. Escitalopram results in a slight decrease in BP without dose effect, though not clinically meaningful. ${ }^{11}$ Furthermore, it has been reported that a modest reduction in SBP and DBP could be observed when statistically comparing fluoxetine with placebo during shortterm treatment. ${ }^{12}$ Effects of venlafaxine and duloxetine on BP have been depicted in previous studies. ${ }^{13-15}$ Nevertheless, whether SSRIs increase or decrease BP during the treatment duration of depression and whether significantly different BP variations exist between SSRIs and SNRIs seem ambiguous. It is reported that SSRIs can affect cardiovascular function. ${ }^{16}$ SSRIs are rarely associated with cardiac death but cause side effects such as hypotension and mild bradycardia. ${ }^{17}$ SNRIs can cause elevation of BP, particularly of DBP. Does antidepressant use act to confound the relationship between psychopathology and BP?

Currently, reports of effects on BP exerted by SSRIs are rather scarce, and there is a lack of detailed analysis investigating effects on BP. Considering that the magnitude of BP changes has not been described elaborately enough with SSRI and SNRI administration, we conducted this meta-analysis in terms of BP change, providing directly perceived clinical evidence. In this context, we reported BP changes during SSRI treatment for depression compared with placebo and SNRIs, respectively.

\section{Materials and methods Data search}

All randomized, double-blind clinical trials which compared SSRIs with SNRIs or placebo in treatment of depression were searched and assessed for inclusion. A computerized search was performed in PubMed, EMBASE, ISI Web of Science, PsycNET, CCRCT, and DARE (up to March 2017) for original research articles. Reference lists of relevant studies and reviews were further examined to reveal additional studies. Search terms included "fluoxetine", "citalopram", "escitalopram", "fluvoxamine", "sertraline", "paroxetine", "placebo", "venlafaxine", "duloxetine", "milnacipran", "major depressive disorder" (MDD), “depression", and "randomized controlled trial" (RCT). The comparison interventions were placebo treatments or SNRIs. Detailed data including changes of SBP and DBP were carefully examined. We excluded open-label trials and studies with insufficient information about BP.

\section{Patients}

The inclusion criteria for patients were: a) outpatients or inpatients meeting the diagnostic standard of MDD according to the Diagnostic and Statistical Manual of Mental Disorders, Fourth Edition (DSM-IV) or text revision (DSM-IV TR) diagnosis of MDD, and b) single episode or recurrent MDD. There was no age restriction. Exclusion criteria were: a) past or current presence of seizure disorder; b) depression with psychotic feature, diagnosis of schizophrenia, schizoaffective disorder, and bipolar disorder; c) posttraumatic stress disorder; d) uncontrolled hypertension; e) female patients who were pregnant or lactating; and f) a history of alcohol or substance dependence or abuse.

\section{Data extraction and quality assessment}

For each trial, we extracted data recorded in a standardized Excel file, including the first author, year of publication, sample size, population age, treatment duration, medication doses, and checked by a third investigator. Two investigators extracted the data and trial quality information from the studies selected for inclusion in the meta-analysis independently to evaluate eligibility. If the studies were approved to meet inclusion criteria by both reviewers, the trials were included in the analysis. Any inconsistencies were reviewed 
and resolved by discussion and consensus. Outcome variables were the effects of individual BP changes.

For each eligible trial, risks of bias were assessed in detail, according to the bias assessment of the Cochrane Handbook for Systematic Reviews of Interventions (version 5.10). Treatment agents, blinding, and randomization were demonstrated in detail according to the primary trials.

\section{Statistical analysis}

We calculated continuous outcomes using weighted mean differences (WMDs) with 95\% CIs, since each study used the same outcome for the studied adverse effects, and this preserves the original BP change, which is intuitively interpreted (eg, a WMD of 5 means a $5 \mathrm{mmHg}$ difference in BP between the two groups). The inverse variance statistical method and random effects model were applied to calculate pooled data. When SDs were not reported, they were derived from other available data or we contacted authors to supply the statistics. In the absence of data from authors, we used the average SDs of other studies with the same medication. ${ }^{18}$

We evaluated study heterogeneity by $\chi^{2}$ test and $I^{2}$ statistics, with statistical significance set at $\chi^{2} P<0.1$ and $I^{2}>50 \%$ indicating heterogeneity. We conducted sensitivity analyses by reestimating pooled WMD by omitting one study at a time to evaluate the influence of each individual study on the overall meta-analysis. Furthermore, for comparing SSRIs with SNRIs, we conducted subgroup analyses based on treatment durations (long-term and short-term) and different ages ( $\geq 18$ years and $<18$ years) to clarify potential sources of heterogeneity.

Publication bias was assessed by conducting Begg's test and Egger's test. If $P r$ or $P$-value was $<0.05$, publication bias of the meta-analysis was considered representative of statistical significance. Data were processed by using the computer program Review Manager (version 5.3. the Nordic Cochrane Centre, Copenhagen, Denmark; The Cochrane Collaboration, 2014) chiefly, and STATA (version 12.0; StataCorp LP, College Station, TX, USA) was used in the quantitative assessment of publication bias and sensitivity analyses as supplement.

\section{Results}

The initial search yielded 1,824 abstracts, of which 628 full texts were inspected, as outlined in Figure 1. There were 23 non-duplicated trials ${ }^{19-41}$ comparing SSRI intervention with placebo or SNRIs included for this meta-analysis, after excluding other interventions and those with lack of analyzable data about BP or length of intervention shorter

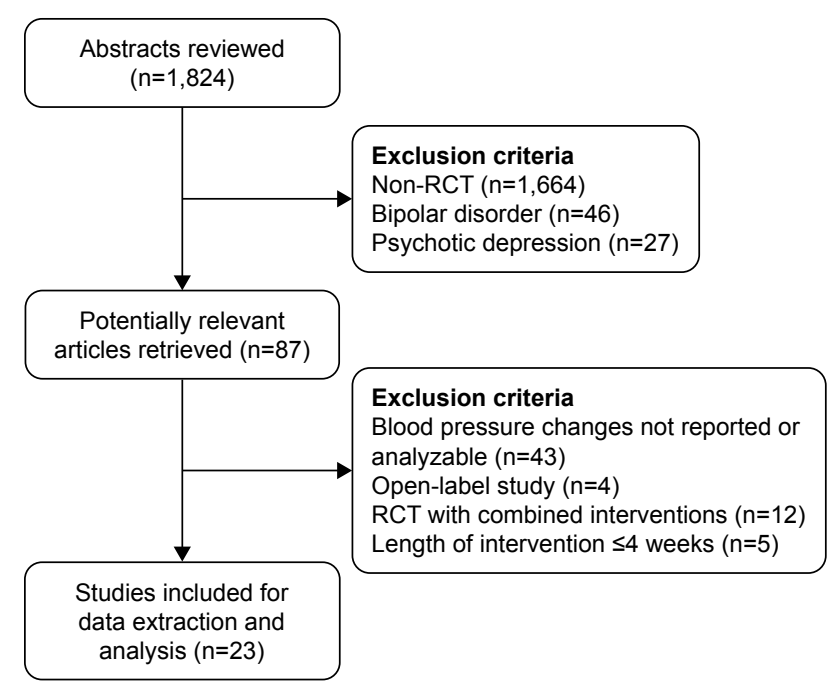

Figure I Flow chart of study selection. Abbreviation: RCT, randomized controlled trial.

than 4 weeks. Except for four studies based on children and teenagers, ${ }^{26,29,30,40}$ all other trials included adults. There were 15 trials available for analysis of comparing SSRIs with placebo. One study included patients with MDD combined with a history of acute myocardial infarction or unstable angina. ${ }^{20}$ Two trials were about MDD combined with coronary artery disease ${ }^{28}$ or depressive disorders combined with acute coronary syndrome, ${ }^{33}$ respectively. Considering the fact that comorbid cardiovascular diseases were in a steady state, antihypertensive and other cardiovascular medications were prescribed on stable doses for study duration, the previously mentioned three trials were included in the analysis. A total of 18 trials comparing SSRIs with two SNRIs were included. No qualified studies on fluvoxamine and milnacipran were identified. There were six trials including different medication doses or durations; thus, those data of identically designed studies were all included in the analysis. In all, the group of SSRIs versus placebo included 4,662 patients and 8,623 patients in the SSRIs versus SNRIs group. Table 1 outlines the main characteristics of the 23 RCTs. Figure 2 presents the summary of the risk of bias of each individual study.

\section{BP changes in SSRI groups versus placebo}

Differences between individual SSRIs and placebo regarding SBP and DBP changes are summarized in Figures 3 and 4. Overall, SSRI interventions were associated with a pooled SBP change of $-0.04 \mathrm{mmHg}(95 \% \mathrm{CI}-0.68,0.59)$ versus placebo (Figure 3), indicating no significant difference with $Z=0.14, P=0.89$. Subgroup difference test revealed no significant difference: $\chi^{2}=3.46, d f=4, P=0.48, I^{2}=0 \%$. 
Table I Characteristics of randomized controlled trials included in the meta-analysis

\begin{tabular}{|c|c|c|c|c|c|c|}
\hline \multirow[t]{2}{*}{ Study } & \multirow[t]{2}{*}{ Design } & \multirow{2}{*}{$\begin{array}{l}\text { Main inclusion } \\
\text { criteria }\end{array}$} & \multirow{2}{*}{$\begin{array}{l}\text { Mean age (SD), years } \\
\text { (SSRI placebo or } \\
\text { SSRIs/placebo/SNRIs) }\end{array}$} & \multirow{2}{*}{$\begin{array}{l}\text { Duration } \\
\text { (weeks) }\end{array}$} & \multicolumn{2}{|c|}{ Intervention, number, and doses } \\
\hline & & & & & Treatment & Comparison \\
\hline $\begin{array}{l}\text { Lenox-Smith } \\
\text { and Jiang } 2008^{19}\end{array}$ & $\begin{array}{l}\text { RCT, } \\
\text { double-blind }\end{array}$ & MDD & $\begin{array}{l}\text { Citalopram } 43 \text { (II.2) } \\
\text { Venlafaxine } 42(10.8)\end{array}$ & 12 & $\begin{array}{l}\text { Citalopram } \\
(20-60 \mathrm{mg} / \mathrm{d}), \mathrm{N}=205\end{array}$ & $\begin{array}{l}\text { Venlafaxine }(75-300 \mathrm{mg} / \mathrm{d}) \text {, } \\
\mathrm{N}=199\end{array}$ \\
\hline $\begin{array}{l}\text { Glassman } \\
\text { et al } 2002^{20}\end{array}$ & $\begin{array}{l}\text { RCT, } \\
\text { double-blind }\end{array}$ & $\begin{array}{l}\text { MDD and AMI } \\
\text { or UA }\end{array}$ & $\begin{array}{l}\text { Sertraline } 56.8(\text { (II.I) } \\
\text { Placebo } 57.6(10.4)\end{array}$ & 16 & $\begin{array}{l}\text { Sertraline } \\
(50-200 \mathrm{mg} / \mathrm{d}), \mathrm{N}=186\end{array}$ & Placebo, $\mathrm{N}=183$ \\
\hline $\begin{array}{l}\text { Nierenberg } \\
\text { et al } 2007^{21}\end{array}$ & $\begin{array}{l}\text { RCT, } \\
\text { double-blind }\end{array}$ & MDD & $\begin{array}{l}\text { Escitalopram } 43.3(13.0) \\
\text { Placebo } 42.5 \text { (I2.3) } \\
\text { Duloxetine } 41.1 \text { (I I.6) }\end{array}$ & 8 & $\begin{array}{l}\text { Escitalopram } \\
(10 \mathrm{mg} / \mathrm{d}), \mathrm{N}=274\end{array}$ & $\begin{array}{l}\text { Duloxetine }(60 \mathrm{mg} / \mathrm{d}) \text {, } \\
\mathrm{N}=273 \text {, placebo, } N=137\end{array}$ \\
\hline $\begin{array}{l}\text { Coleman et al } \\
200 I^{22}\end{array}$ & $\begin{array}{l}\text { RCT, } \\
\text { double-blind }\end{array}$ & $\begin{array}{l}\text { Major } \\
\text { depression }\end{array}$ & $\begin{array}{l}\text { Fluoxetine } 37.1(18-76)^{\mathrm{a}} \\
\text { Placebo } 36.7(19-62)\end{array}$ & 8 & $\begin{array}{l}\text { Fluoxetine } \\
(20-60 \mathrm{mg} / \mathrm{d}), \mathrm{N}=154\end{array}$ & Placebo, $N=152$ \\
\hline $\begin{array}{l}\text { Nemeroff et al } \\
2007^{23}\end{array}$ & $\begin{array}{l}\text { RCT, } \\
\text { double-blind }\end{array}$ & MDD & $\begin{array}{l}\text { Fluoxetine } 37.9 \text { (I I.5) } \\
\text { Placebo } 40.4 \text { (I I.7) } \\
\text { Venlafaxine } 40.1 \text { (I I.I) }\end{array}$ & 6 & $\begin{array}{l}\text { Fluoxetine } \\
(20-60 \mathrm{mg} / \mathrm{d}), \mathrm{N}=101\end{array}$ & $\begin{array}{l}\text { Venlafaxine }(75-225 \mathrm{mg} / \mathrm{d}) \text {, } \\
\mathrm{N}=96 \text {, placebo, } \mathrm{N}=101\end{array}$ \\
\hline $\begin{array}{l}\text { Oslin et al } \\
2003^{24}\end{array}$ & $\begin{array}{l}\text { RCT, } \\
\text { double-blind }\end{array}$ & $\begin{array}{l}\text { Depressive } \\
\text { disorder }\end{array}$ & $\begin{array}{l}\text { Sertraline } 83.8(9.8) \\
\text { Venlafaxine } 81.2(10.8)\end{array}$ & 10 & $\begin{array}{l}\text { Sertraline } \\
(25-100 \mathrm{mg} / \mathrm{d}), \mathrm{N}=25\end{array}$ & $\begin{array}{l}\text { Venlafaxine }(75-150 \mathrm{mg} / \mathrm{d}), \\
\mathrm{N}=27\end{array}$ \\
\hline $\begin{array}{l}\text { Goldstein et al } \\
2004^{25}\end{array}$ & $\begin{array}{l}\text { RCT, } \\
\text { double-blind }\end{array}$ & Depression & $\begin{array}{l}\text { Paroxetine } 40(\mathrm{II}) \\
\text { Placebo } 57(64) \\
\text { Duloxetine }(40 \mathrm{mg} / \mathrm{d}) 48(56) \\
\text { Duloxetine }(80 \mathrm{mg} / \mathrm{d}) 56(62)\end{array}$ & 8 & $\begin{array}{l}\text { Paroxetine } \\
(20 \mathrm{mg} / \mathrm{d}), \mathrm{N}=87\end{array}$ & $\begin{array}{l}\text { Duloxetine }(40 \mathrm{mg} / \mathrm{d}) \\
\mathrm{N}=86 \\
\text { Duloxetine }(80 \mathrm{mg} / \mathrm{d}) \\
\mathrm{N}=91 \text {, placebo, } \mathrm{N}=89\end{array}$ \\
\hline $\begin{array}{l}\text { Brent et al } \\
2008^{26}\end{array}$ & $\begin{array}{l}\text { RCT, } \\
\text { double-blind }\end{array}$ & MDD & $\begin{array}{l}\text { Different SSRIs I6.0(I.6) } \\
\text { Venlafaxine I5.8 (I.5) }\end{array}$ & 12 & $\begin{array}{l}\text { Different SSRIs } \\
\text { (paroxetine, } \\
\text { citalopram, fluoxetine), } \\
(20-40 \mathrm{mg} / \mathrm{d}), \mathrm{N}=168\end{array}$ & $\begin{array}{l}\text { Venlafaxine } \\
\text { (150-225 mg/d), N=166 }\end{array}$ \\
\hline $\begin{array}{l}\text { Sheehan et al } \\
2009^{27}\end{array}$ & $\begin{array}{l}\text { RCT, } \\
\text { double-blind }\end{array}$ & MDD & $\begin{array}{l}\text { Fluoxetine } 37.8(\mathrm{II} .1) \\
\text { Placebo } 39.9(13.0) \\
\text { Venlafaxine } 41.7(12.8)\end{array}$ & 6 & $\begin{array}{l}\text { Fluoxetine } \\
(60-80 \mathrm{mg} / \mathrm{d}), \mathrm{N}=99\end{array}$ & $\begin{array}{l}\text { Venlafaxine } \\
(225-375 \mathrm{mg} / \mathrm{d}), \mathrm{N}=94, \\
\text { placebo, } \mathrm{N}=95\end{array}$ \\
\hline $\begin{array}{l}\text { Lesperance } \\
\text { et al } 2007^{28}\end{array}$ & $\begin{array}{l}\text { RCT, } \\
\text { double-blind }\end{array}$ & MDD and CAD & $\begin{array}{l}\text { Citalopram } 57.9(9.15) \\
\text { Placebo } 58.4(9.16)\end{array}$ & 12 & $\begin{array}{l}\text { Citalopram } \\
(20-40 \mathrm{mg} / \mathrm{d}), \mathrm{N}=142\end{array}$ & Placebo, $\mathrm{N}=142$ \\
\hline $\begin{array}{l}\text { Emslie et al } \\
2014^{29}\end{array}$ & $\begin{array}{l}\text { RCT, } \\
\text { double-blind }\end{array}$ & MDD & $\begin{array}{l}\text { Fluoxetine I } 3.0(3.2) \\
\text { Placebo I } 3.1(2.9) \\
\text { Duloxetine }(60 \mathrm{mg} / \mathrm{d}) \\
\text { I } 2.9(2.9) \\
\text { Duloxetine }(30 \mathrm{mg} / \mathrm{d}) \\
\text { I } 2.9(2.9)\end{array}$ & 10 & $\begin{array}{l}\text { Fluoxetine } \\
(20-40 \mathrm{mg} / \mathrm{d}), \mathrm{N}=\mathrm{I} / 2\end{array}$ & $\begin{array}{l}\text { Duloxetine }(60 \mathrm{mg} / \mathrm{d}) \text {, } \\
\mathrm{N}=105, \\
\text { Duloxetine }(30 \mathrm{mg} / \mathrm{d}) \text {, } \\
\mathrm{N}=1 \mid \mathrm{4}, \\
\text { placebo, } \mathrm{N}=117\end{array}$ \\
\hline $\begin{array}{l}\text { Emslie et al } \\
2015^{30}\end{array}$ & $\begin{array}{l}\text { RCT, } \\
\text { double-blind }\end{array}$ & MDD & $13.1^{\mathrm{b}}$ & 10 & $\begin{array}{l}\text { Fluoxetine } \\
(20-40 \mathrm{mg} / \mathrm{d}), \mathrm{N}=226\end{array}$ & $\begin{array}{l}\text { Duloxetine }(60-120 \mathrm{mg} / \mathrm{d}) \text {, } \\
\mathrm{N}=332 \text {, placebo, } \mathrm{N}=220\end{array}$ \\
\hline $\begin{array}{l}\text { Croft et al } \\
\mid 999^{31}\end{array}$ & $\begin{array}{l}\text { RCT, } \\
\text { double-blind }\end{array}$ & MDD & $\begin{array}{l}\text { Sertraline } 36.0(19-61)^{c} \\
\text { Placebo } 37.4(19-64)\end{array}$ & 8 & $\begin{array}{l}\text { Sertraline } \\
(50-200 \mathrm{mg} / \mathrm{d}), N=119\end{array}$ & Placebo, $\mathrm{N}=121$ \\
\hline $\begin{array}{l}\text { Nelson et al } \\
2006^{32}\end{array}$ & $\begin{array}{l}\text { RCT, } \\
\text { double-blind }\end{array}$ & MDD & $\begin{array}{l}\text { Paroxetine } 43.2 \text { (I I.9) } \\
\text { Placebo } 42.9 \text { (I } 2.5) \\
\text { Duloxetine } 43.4 \text { (I } 2.2)\end{array}$ & 8 & $\begin{array}{l}\text { Paroxetine } \\
(20 \mathrm{mg} / \mathrm{d}), \mathrm{N}=359\end{array}$ & $\begin{array}{l}\text { Duloxetine }(40-120 \mathrm{mg} / \mathrm{d}) \text {, } \\
\mathrm{N}=736, \\
\text { placebo, } \mathrm{N}=37 \mathrm{I}\end{array}$ \\
\hline $\begin{array}{l}\text { Kim et al } \\
2015^{33}\end{array}$ & $\begin{array}{l}\text { RCT, } \\
\text { double-blind }\end{array}$ & $\begin{array}{l}\text { Depressive } \\
\text { disorders and } \\
\text { ACS }\end{array}$ & $\begin{array}{l}\text { Escitalopram 60.1 (10.9) } \\
\text { Placebo } 58.5 \text { (10.6) }\end{array}$ & 24 & $\begin{array}{l}\text { Escitalopram } \\
(5-20 \mathrm{mg} / \mathrm{d}), \mathrm{N}=108\end{array}$ & Placebo, $\mathrm{N}=109$ \\
\hline $\begin{array}{l}\text { Keller et al } \\
2007^{34}\end{array}$ & $\begin{array}{l}\text { RCT, } \\
\text { double-blind }\end{array}$ & MDD & $\begin{array}{l}10 \text { w: Fluoxetine } 40.0 \text { ( } 11.6) \\
\text { Venlafaxine } 39.6 \text { (12.2) } \\
34 \text { w: Fluoxetine } 40.9 \text { (I I.5) } \\
\text { Venlafaxine } 40.4 \text { (12.0) }\end{array}$ & $\begin{array}{l}10 \\
34\end{array}$ & $\begin{array}{l}\text { Fluoxetine } \\
(20-60 \mathrm{mg} / \mathrm{d}) \\
(10 \mathrm{w}, \mathrm{N}=266) \\
(34 \mathrm{w}, \mathrm{N}=185)\end{array}$ & $\begin{array}{l}\text { Venlafaxine }(75-300 \mathrm{mg} / \mathrm{d}), \\
(10 \mathrm{w}, \mathrm{N}=78 \mathrm{I}) \\
(34 \mathrm{w}, \mathrm{N}=530)\end{array}$ \\
\hline $\begin{array}{l}\text { Detke et al } \\
2004^{35}\end{array}$ & $\begin{array}{l}\text { RCT, } \\
\text { double-blind }\end{array}$ & MDD & $\begin{array}{l}\text { Paroxetine } 42.0(10.6) \\
\text { Placebo } 43.7(12.2) \\
\text { Duloxetine }(80 \mathrm{mg} / \mathrm{d}) \\
43.1(11.1) \\
\text { Duloxetine }(120 \mathrm{mg} / \mathrm{d}) \\
44.7(10.7)\end{array}$ & $\begin{array}{l}8 \\
24\end{array}$ & $\begin{array}{l}\text { Paroxetine } \\
(20 \mathrm{mg} / \mathrm{d}) \\
(8 \mathrm{w}, \mathrm{N}=85 ; 24 \\
\mathrm{w}, \mathrm{N}=70)\end{array}$ & $\begin{array}{l}\text { Duloxetine }(80 \mathrm{mg} / \mathrm{d}), \\
(8 \mathrm{w}, \mathrm{N}=93 ; 24 \mathrm{w}, \mathrm{N}=70) \\
\text { Duloxetine }(120 \mathrm{mg} / \mathrm{d}), \\
(8 \mathrm{w}, \mathrm{N}=93 ; 24 \mathrm{w}, \mathrm{N}=74) \\
\text { placebo }(8 \mathrm{w}, \mathrm{N}=93 ; 24 \mathrm{w}, \\
\mathrm{N}=58)\end{array}$ \\
\hline $\begin{array}{l}\text { Allard et al } \\
2004^{36}\end{array}$ & $\begin{array}{l}\text { RCT, } \\
\text { double-blind }\end{array}$ & $\begin{array}{l}\text { Major } \\
\text { depression }\end{array}$ & $\begin{array}{l}\text { Citalopram } 72.5(5.7) \\
\text { Venlafaxine } 73.6(5.9)\end{array}$ & $\begin{array}{l}8 \\
22\end{array}$ & $\begin{array}{l}\text { Citalopram } \\
(10-20 \mathrm{mg} / \mathrm{d}), \mathrm{N}=75\end{array}$ & $\begin{array}{l}\text { Venlafaxine }(75-150 \mathrm{mg} / \mathrm{d}) \text {, } \\
\mathrm{N}=73\end{array}$ \\
\hline
\end{tabular}


Table I (Continued)

\begin{tabular}{|c|c|c|c|c|c|c|}
\hline \multirow[t]{2}{*}{ Study } & \multirow[t]{2}{*}{ Design } & \multirow{2}{*}{$\begin{array}{l}\text { Main inclusion } \\
\text { criteria }\end{array}$} & \multirow{2}{*}{$\begin{array}{l}\text { Mean age (SD), years } \\
\text { (SSRI Placebo or } \\
\text { SSRIs/Placebo/SNRIs) }\end{array}$} & \multirow{2}{*}{$\begin{array}{l}\text { Duration } \\
\text { (weeks) }\end{array}$} & \multicolumn{2}{|c|}{ Intervention, number, and doses } \\
\hline & & & & & Treatment & Comparison \\
\hline $\begin{array}{l}\text { Perahia et al } \\
2006^{37}\end{array}$ & $\begin{array}{l}\text { RCT, } \\
\text { double-blind }\end{array}$ & MDD & $\begin{array}{l}\text { Paroxetine } 45.8(10.6) \\
\text { Placebo } 44.7(10.1) \\
\text { Duloxetine }(80 \mathrm{mg} / \mathrm{d}) \\
46.5(12.7) \\
\text { Duloxetine }(120 \mathrm{mg} / \mathrm{d}) \\
44.0(10.8)\end{array}$ & $\begin{array}{l}8 \\
32\end{array}$ & $\begin{array}{l}\text { Paroxetine } \\
(20 \mathrm{mg} / \mathrm{d}),(8 \mathrm{w}, \\
\mathrm{N}=96 ; 32 \mathrm{w}, \mathrm{N}=70)\end{array}$ & $\begin{array}{l}\text { Duloxetine }(80 \mathrm{mg} / \mathrm{d}), \\
(8 \mathrm{w}, \mathrm{N}=93 ; 32 \mathrm{w}, \mathrm{N}=70) \\
\text { Duloxetine }(120 \mathrm{mg} / \mathrm{d}), \\
(8 \mathrm{w}, \mathrm{N}=102 ; 32 \mathrm{w}, \mathrm{N}=80) \\
\text { placebo }(8 \mathrm{w}, \mathrm{N}=99 ; 24 \mathrm{w}, \\
\mathrm{N}=70)\end{array}$ \\
\hline Lee et al $2007^{38}$ & $\begin{array}{l}\text { RCT, } \\
\text { double-blind }\end{array}$ & MDD & $\begin{array}{l}\text { Paroxetine } 38.0 \text { (I5.27) } \\
\text { Duloxetine } 39.0 \text { (13.95) }\end{array}$ & 8 & $\begin{array}{l}\text { Paroxetine } \\
(20 \mathrm{mg} / \mathrm{d}), \mathrm{N}=240\end{array}$ & $\begin{array}{l}\text { Duloxetine }(60 \mathrm{mg} / \mathrm{d}) \text {, } \\
\mathrm{N}=238\end{array}$ \\
\hline $\begin{array}{l}\text { Bielski et al } \\
2004^{39}\end{array}$ & $\begin{array}{l}\text { RCT, } \\
\text { double-blind }\end{array}$ & MDD & $\begin{array}{l}\text { Escitalopram } 37.3 \text { (12.3) } \\
\text { Venlafaxine } 37.5 \text { (II.6) }\end{array}$ & 8 & $\begin{array}{l}\text { Escitalopram } \\
(20 \mathrm{mg} / \mathrm{d}), \mathrm{N}=97\end{array}$ & $\begin{array}{l}\text { Venlafaxine }(225 \mathrm{mg} / \mathrm{d}) \text {, } \\
\mathrm{N}=98\end{array}$ \\
\hline $\begin{array}{l}\text { Atkinson et al } \\
2014^{40}\end{array}$ & $\begin{array}{l}\text { RCT, } \\
\text { double-blind }\end{array}$ & MDD & $\begin{array}{l}\text { Fluoxetine I3.I (3.3) } \\
\text { Placebo I } 3.3(3.1) \\
\text { Duloxetine I3.I (3.0) }\end{array}$ & 10 & $\begin{array}{l}\text { Fluoxetine } \\
(20-40 \mathrm{mg} / \mathrm{d}), \mathrm{N}=1 \mathrm{l} 4\end{array}$ & $\begin{array}{l}\text { Duloxetine }(60-120 \mathrm{mg} / \mathrm{d}) \text {, } \\
\mathrm{N}=1 / 13 \text {, placebo, } \mathrm{N}=103\end{array}$ \\
\hline $\begin{array}{l}\text { Wade et al } \\
2007^{41}\end{array}$ & $\begin{array}{l}\text { RCT, } \\
\text { double-blind }\end{array}$ & MDD & $\begin{array}{l}\text { Escitalopram } 43.3 \text { (I I.6) } \\
\text { Duloxetine } 44.5 \text { (I I .0) }\end{array}$ & 24 & $\begin{array}{l}\text { Escitalopram } \\
(20 \mathrm{mg} / \mathrm{d}), \mathrm{N}=1 / 2\end{array}$ & $\begin{array}{l}\text { Duloxetine }(60 \mathrm{mg} / \mathrm{d}) \text {, } \\
\mathrm{N}=1 / 4\end{array}$ \\
\hline
\end{tabular}

Notes: aSDs were missing. Age ranges of fluoxetine group and placebo were 18-76 and 19-62 years, respectively. ${ }^{\mathrm{b}}$ The mean age of randomized patients included in the three groups was 13.1. 'SDs were missing. Age ranges of sertraline group and placebo were 19-6I and 19-64 years, respectively.

Abbreviations: RCT, randomized controlled trial; MDD, major depressive disorder; SSRI, selective serotonin reuptake inhibitor; SNRI, serotonin and noradrenaline reuptake inhibitor; AMI, acute myocardial infarction; UA, unstable angina; CAD, coronary artery disease; ACS, acute coronary syndrome.

A pooled DBP change in SSRIs versus placebo group was $0.08 \mathrm{mmHg}(95 \% \mathrm{CI}-0.43,0.60)$, with no statistical significance, $Z=0.32, P=0.75$ (Figure 4 ). There was no significant difference in DBP changes among subgroups: $\chi^{2}=1.01, d f=4$, $P=0.91, I^{2}=0 \%$.

\section{BP changes in SSRIs versus SNRIs groups}

Twenty-eight studies reported BP changes in SSRIs versus SNRIs. In the pooled analysis, overall SBP changes and DBP changes revealed statistically significant differences (WMD -1.5 mmHg, 95\% CI -2.15, -0.84, $Z=4.46$, $P<0.00001$, Figure 5, and WMD $-1.34 \mathrm{mmHg}, 95 \%$ CI $-1.92,-0.75, Z=6.18, P<0.00001$, Figure 6). There was a low level of heterogeneity across all studies in SBP changes $\left(I^{2}=39 \%, P=0.02\right.$, Figure 5$)$, while a medium level of heterogeneity was detected in DBP changes $\left(I^{2}=54 \%\right.$, $P=0.0004$, Figure 6).

\section{Short-/long-term duration}

SBP changes between SSRIs and SNRIs in shortterm duration ( $\leq 8$ weeks) and long-term duration $(>8$ weeks) differed statistically (WMD $-1.51 \mathrm{mmHg}, 95 \%$ $\mathrm{CI}-2.44,-0.58, Z=3.18, P=0.001$ and $\mathrm{WMD}-1.46 \mathrm{mmHg}$, $95 \%$ CI $-2.42,-0.49, Z=2.95, P=0.003$ ) (Figure 5). Significant differences were observed in DBP changes in short-/long term duration (WMD $-1.10 \mathrm{mmHg}, 95 \% \mathrm{CI}-1.82,-0.39$, $Z=3.04, P=0.002$ and $\mathrm{WMD}-1.49 \mathrm{mmHg}, 95 \% \mathrm{CI}-2.37$, $-0.61, Z=3.31, P=0.0009$ ) (Figure 6). Tests for subgroup differences of SBP and DBP changes revealed no significant difference: $\chi^{2}=0.01, d f=1, P=0.94, I^{2}=0 \%$ and $\chi^{2}=0.44, d f=1$, $P=0.51, I^{2}=0 \%$.

\section{Different ages}

A significant difference in SBP changes resulting from age ( $<18$ or $\geq 18$ years) was observed when comparing SSRIs with SNRIs (WMD - 1.31 mmHg, 95\% $\mathrm{CI}-2.31,-0.31, Z=2.56, P=0.01$ and $\mathrm{WMD}-1.51 \mathrm{mmHg}$, 95\% CI $-2.31,-0.70, Z=3.68, P=0.0002$ ) (Figure 7). DBP changes resulting from age between SSRIs and SNRIs showed a statistical difference (WMD $-1.84 \mathrm{mmHg}, 95 \%$ $\mathrm{CI}-3.66,-0.01, Z=1.97, P=0.05$ and $\mathrm{WMD}-1.24 \mathrm{mmHg}$, $95 \% \mathrm{CI}-1.85,-0.63, Z=3.96, P<0.0001$ ) (Figure 8). Tests for subgroup differences of SBP and DBP changes revealed no significant difference: $\chi^{2}=0.09, d f=1, P=0.76, I^{2}=0 \%$ and $\chi^{2}=0.37, d f=1, P=0.54, I^{2}=0 \%$.

\section{Publication bias and sensitivity analysis}

We assessed the possibility of publication bias in the articles, which compared the effects on BP in groups of SSRIs versus placebo and SSRIs versus SNRIs. The funnel plot of Begg illustrated a symmetrical distribution of the points, suggesting a lack of publication bias. No obvious publication bias was found by Begg's test and Egger's test (Figure 9). Sensitivity analyses by reestimating pooled WMD when excluding one study at a time showed similar results (Figures 10 and 11). 


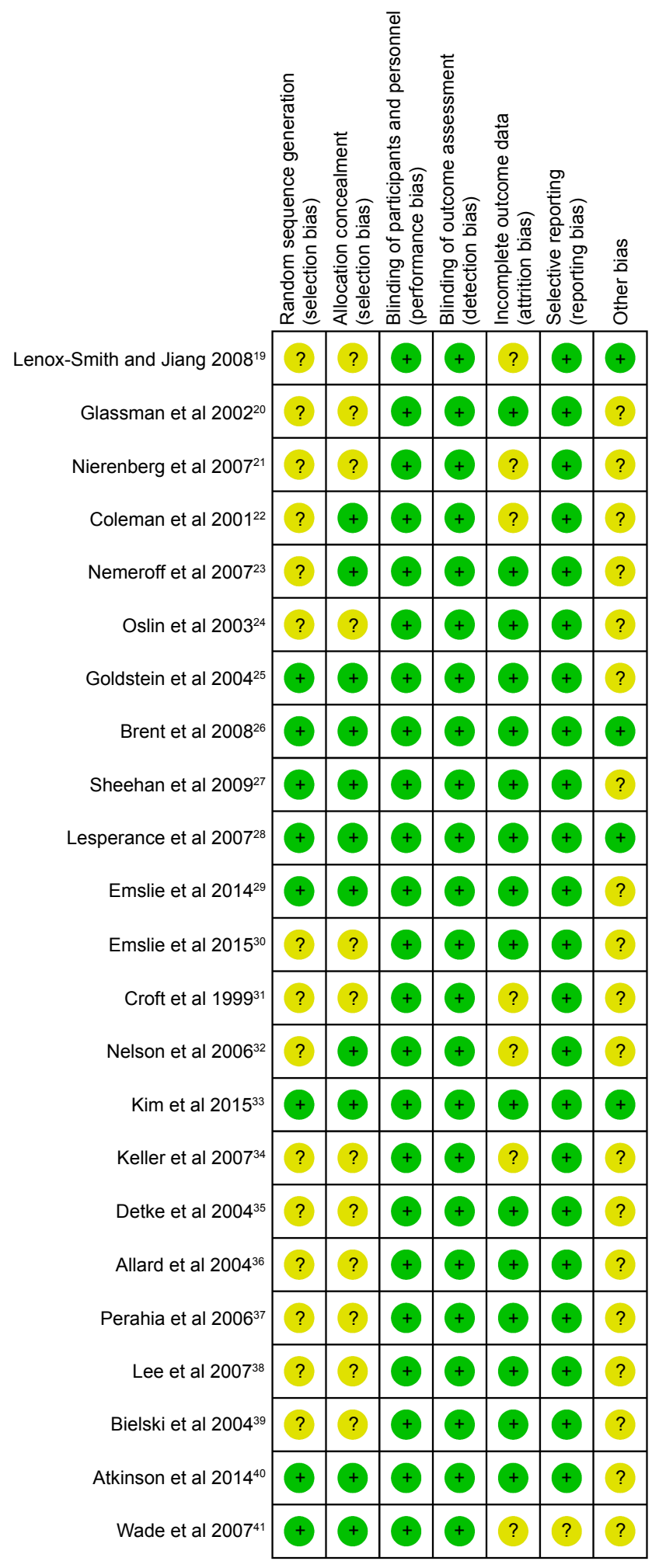

Figure 2 Assessment of risk of bias for each individual trial. ?, unclear risk of bias; + , low risk of bias.

\section{Discussion}

The main purpose of this meta-analysis was to critically evaluate the effects of SSRIs and SNRIs on BP in quantification. As illustrated in our randomized, double blind meta-analysis, WMDs varied little among five kinds of SSRIs when compared with placebo. A test on the subgroups revealed no statistical difference, indicating little difference in BP among the five kinds of SSRIs. SSRIs might not cause more apparent fluctuation of BP than placebo. In terms of definite BP changes, each SSRI may be associated with $<3 \mathrm{mmHg}$ variation during the period of trials, which showed no close connection with management of $\mathrm{BP}$, at the same time suggesting that SSRIs might be safe in regard to BP. Furthermore, it is reported that depressive disorder is associated with average lower levels of SBP and $\mathrm{DBP}^{6}$ and less hypertension. ${ }^{10}$

With regard to changes in BP caused by SSRIs versus SNRIs, WMDs were significantly different, though the exact numerical values were small with variations of $<3 \mathrm{mmHg}$. A conclusion that fluctuations in BP were not significantly different in groups of SSRIs compared with placebo may be drawn from our meta-analysis, it may be inferred that SNRIs could lead to higher BP than SSRIs. Both in short-term and long-term duration, SNRIs were associated with escalation of SBP and DBP to some extent. These findings are consistent with conclusions drawn in the previous reports focusing on venlafaxine. ${ }^{13,14}$ Mean $\mathrm{BP}^{13}$ and supine $\mathrm{DBP}^{14}$ increase with incremental doses of venlafaxine. Incidence of clinically significant increases in BP can be lower at doses below $200 \mathrm{mg}$ daily, ${ }^{13}$ and only doses above $300 \mathrm{mg} /$ day lead to statistical and clinical significance of the incidence of elevated supine DBP. ${ }^{14}$ A previous study reported that duloxetine at supratherapeutic doses increases supine SBP and DBP by maxima of $\sim 12 \mathrm{mmHg}$ and $7 \mathrm{mmHg}$ above baseline, respectively. ${ }^{15}$ Doses of venlafaxine and duloxetine in the included trials of our analysis varied between $75-375 \mathrm{mg} / \mathrm{d}$ and $40-120$ $\mathrm{mg} / \mathrm{d}$, respectively. Because of the paucity of eligible studies and dose modulations, analysis for different doses of medications was not conducted. Nevertheless, it is reliable to highlight that greater BP changes are associated with SNRIs compared to SSRIs; thus, clinicians should monitor BP periodically throughout treatment with venlafaxine and duloxetine.

Depression is associated with reductions in heart rate variability ${ }^{42}$ and might be related to low cardiac vagal control, ${ }^{43}$ as well as central autonomic dysfunction, a shift of autonomic balance toward sympathetic predominance, leading to cardiovascular somatic symptoms of depression such as higher heart rate and BP lability. ${ }^{11,42,44}$ Some studies support the hypothesis that depression is associated with lower BP. ${ }^{46,43}$ However, how BP changes when depressive symptoms are ameliorated still remains obscure. Antidepressant agents, such as SSRIs and SNRIs, 


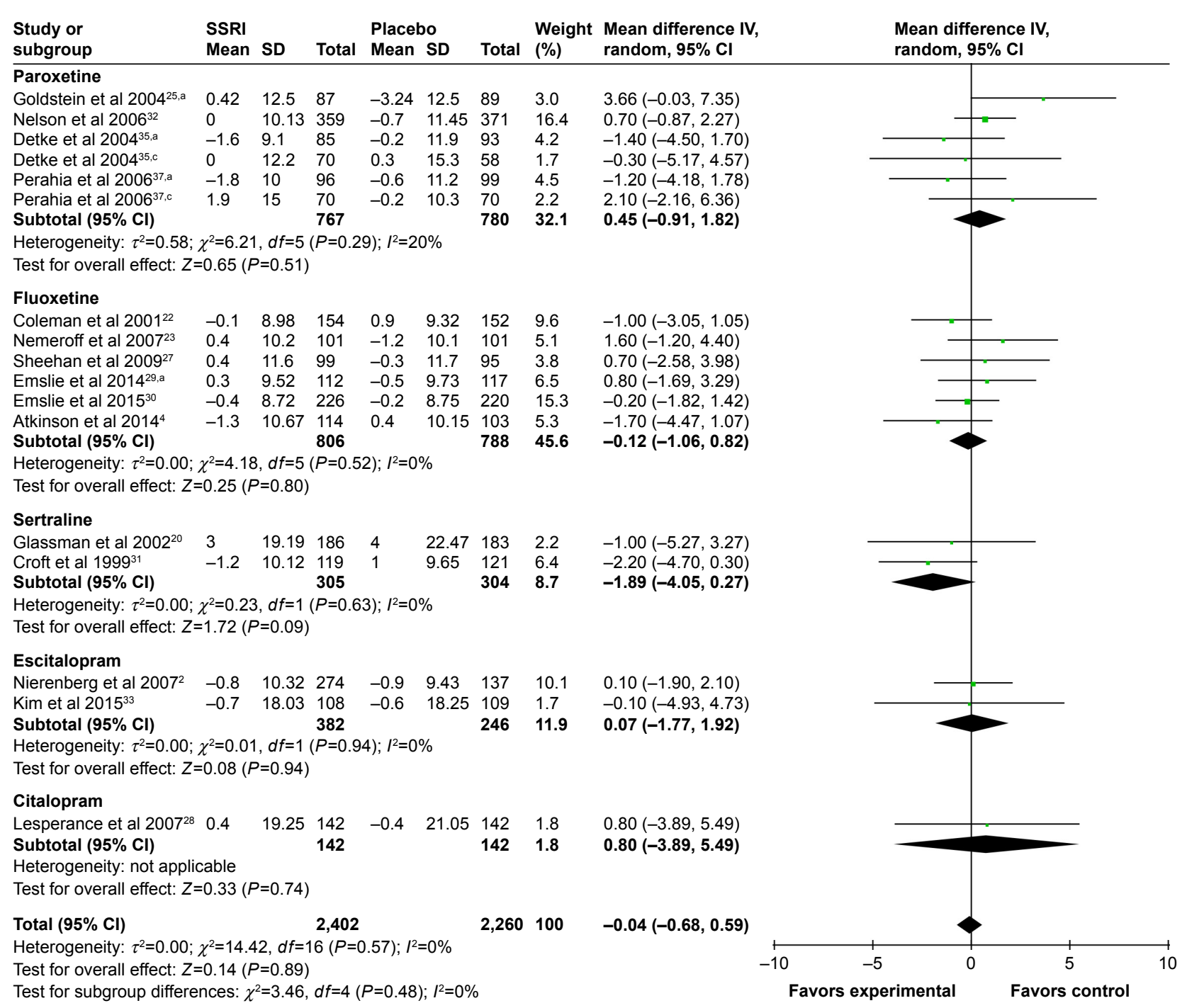

Figure 3 A forest plot of RCTs comparing SSRI group with placebo group for change in systolic blood pressure. Abbreviations: RCTs, randomized controlled trials; SSRI, selective serotonin reuptake inhibitor.

\begin{tabular}{|c|c|c|c|c|c|c|c|c|c|}
\hline \multirow{2}{*}{$\begin{array}{l}\text { Study or } \\
\text { subgroup } \\
\text { Paroxetine }\end{array}$} & \multirow[t]{2}{*}{$\begin{array}{l}\text { SSRI } \\
\text { Mean }\end{array}$} & \multirow[t]{2}{*}{ SD } & \multirow[t]{2}{*}{ Total } & \multirow{2}{*}{\multicolumn{2}{|c|}{$\begin{array}{l}\text { Placebo } \\
\text { Mean SD }\end{array}$}} & \multirow[t]{2}{*}{ Total } & \multirow[t]{2}{*}{$\begin{array}{l}\text { Weight } \\
(\%)\end{array}$} & \multirow[t]{2}{*}{$\begin{array}{l}\text { Mean difference IV, } \\
\text { random, } 95 \% \mathrm{CI}\end{array}$} & $\begin{array}{l}\text { Mean difference IV, } \\
\text { random, } 95 \% \mathrm{CI}\end{array}$ \\
\hline & & & & & & & & & \\
\hline Goldstein et al $2004^{25, a}$ & 0.34 & 9.97 & 87 & -0.47 & 8.61 & 89 & 3.5 & $0.81(-1.94,3.56)$ & \\
\hline Nelson et al $2006^{32}$ & 0.5 & 9.62 & 359 & 0 & 8.45 & 371 & 15.1 & $0.50(-0.82,1.82)$ & \\
\hline Detke et al $2004^{35, a}$ & -2.1 & 8.8 & 85 & -0.3 & 8 & 93 & 4.3 & $-1.80(-4.28,0.68)$ & \\
\hline Detke et al $2004^{35, c}$ & 0.2 & 8.8 & 70 & -0.9 & 9.3 & 58 & 2.7 & $1.10(-2.06,4.26)$ & \\
\hline Perahia et al $2006^{37, a}$ & -0.4 & 6.7 & 96 & 0.7 & 8.2 & 99 & 6.0 & $-1.10(-3.20,1.00)$ & \\
\hline Perahia et al $2006^{37, c}$ & 0.6 & 8.7 & 70 & -0.2 & 7.5 & 70 & 3.6 & $0.80(-1.89,3.49)$ & \\
\hline Subtotal $(95 \% \mathrm{Cl})$ & & & 767 & & & 780 & 35.2 & $0.06(-0.81,0.92)$ & \\
\hline \multirow{2}{*}{\multicolumn{9}{|c|}{$\begin{array}{l}\text { Heterogeneity: } \tau^{2}=0.00 ; \chi^{2}=4.76, d f=5(P=0.45) ; I^{2}=0 \% \\
\text { Test for overall effect: } Z=0.13(P=0.90)\end{array}$}} & \\
\hline & & & & & & & & & \\
\hline \multicolumn{10}{|l|}{ Fluoxetine } \\
\hline Coleman et al $2001^{22}$ & 0.3 & 7.86 & 154 & 1 & 8.54 & 152 & 7.8 & $-0.70(-2.54,1.14)$ & \\
\hline Nemeroff et al $2007^{23}$ & 0.2 & 7.7 & 101 & -1.3 & 7.5 & 101 & 6.0 & $1.50(-0.60,3.60)$ & \\
\hline Sheehan et al $2009^{27}$ & 2.2 & 8.8 & 99 & 0.9 & 8.5 & 95 & 4.5 & $1.30(-1.13,3.73)$ & \\
\hline Emslie et al $2014^{29, a}$ & 1.7 & 8.47 & 112 & 0.7 & 8.65 & 117 & 5.4 & $1.00(-1.22,3.22)$ & \\
\hline Emslie et al $2015^{30}$ & 0.2 & 7.82 & 226 & 0.3 & 7.71 & 220 & 12.6 & $-0.10(-1.54,1.34)$ & \\
\hline Atkinson et al $2014^{4}$ & -1.4 & 8.54 & 114 & 0.2 & 9.13 & 103 & 4.7 & $-1.60(-3.96,0.76)$ & - \\
\hline Subtotal $(95 \% \mathrm{Cl})$ & & & 806 & & & 788 & 40.9 & $0.16(-0.73,1.06)$ & \\
\hline \multirow{2}{*}{\multicolumn{9}{|c|}{$\begin{array}{l}\text { Heterogeneity: } \tau^{2}=0.22 ; \chi^{2}=6.06, d f=5(P=0.30) ; I^{2}=18 \% \\
\text { Test for overall effect: } Z=0.36(P=0.72)\end{array}$}} & \\
\hline & & & & & & & & & \\
\hline
\end{tabular}

Figure 4 (Continued) 


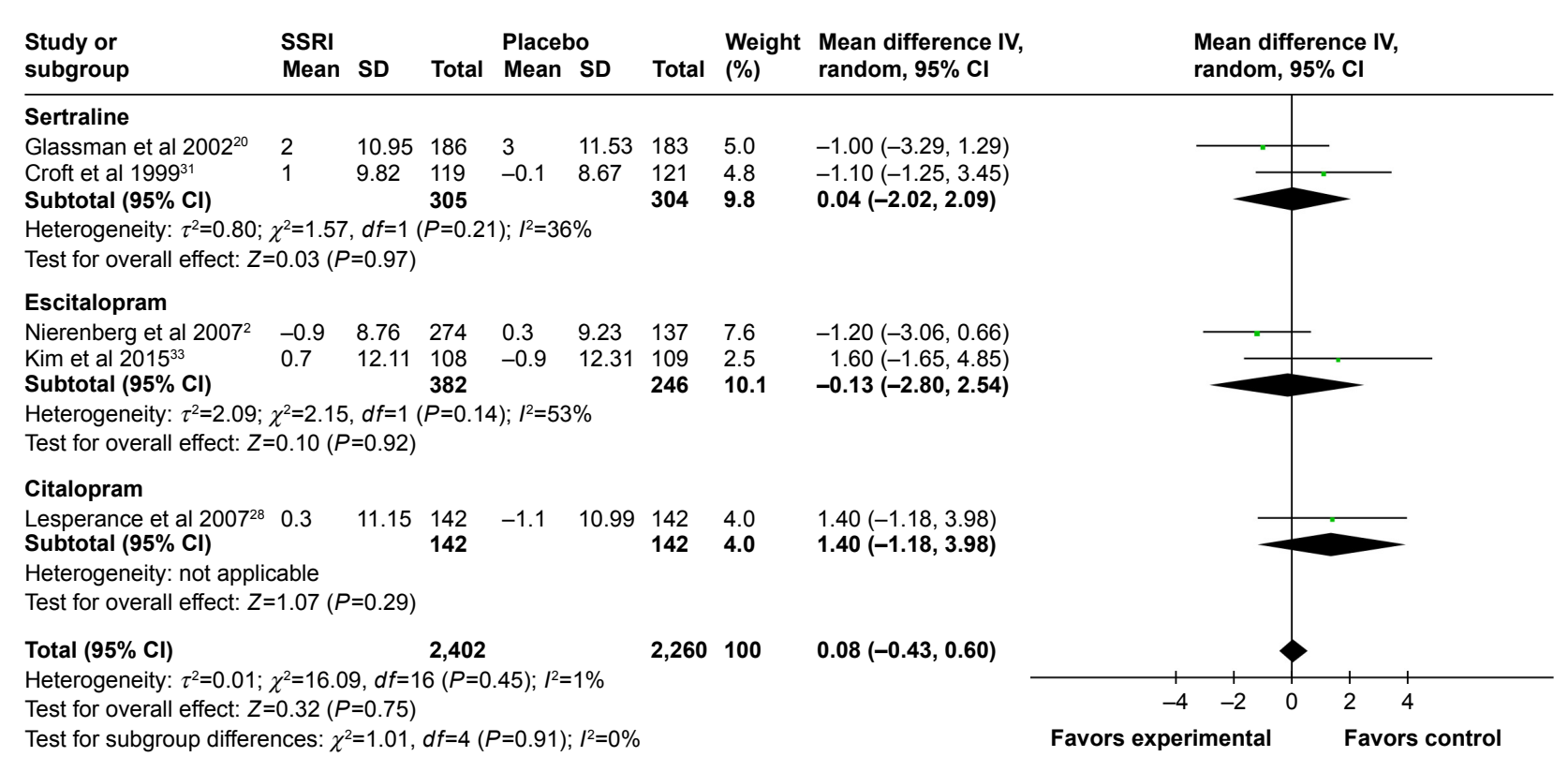

Figure 4 A forest plot of RCTs comparing SSRI group with placebo group for change in diastolic blood pressure.

Abbreviations: RCTs, randomized controlled trials; SSRI, selective serotonin reuptake inhibitor.

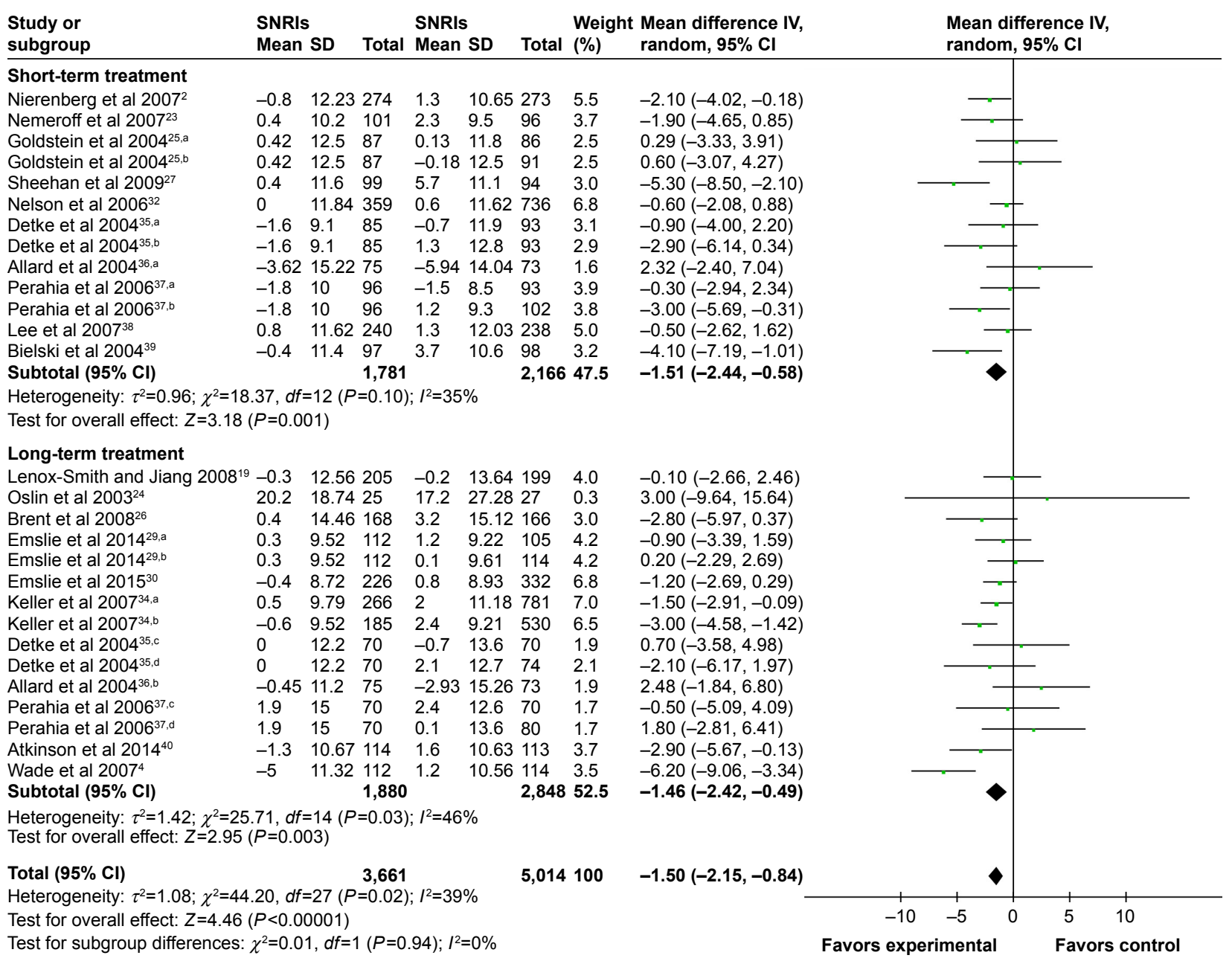

Figure 5 A forest plot of RCTs comparing SSRI group with SNRI group for change in systolic blood pressure change of short-/long-term duration. Abbreviations: RCTs, randomized controlled trials; SSRI, selective serotonin reuptake inhibitor; SNRI, serotonin and noradrenaline reuptake inhibitor. 


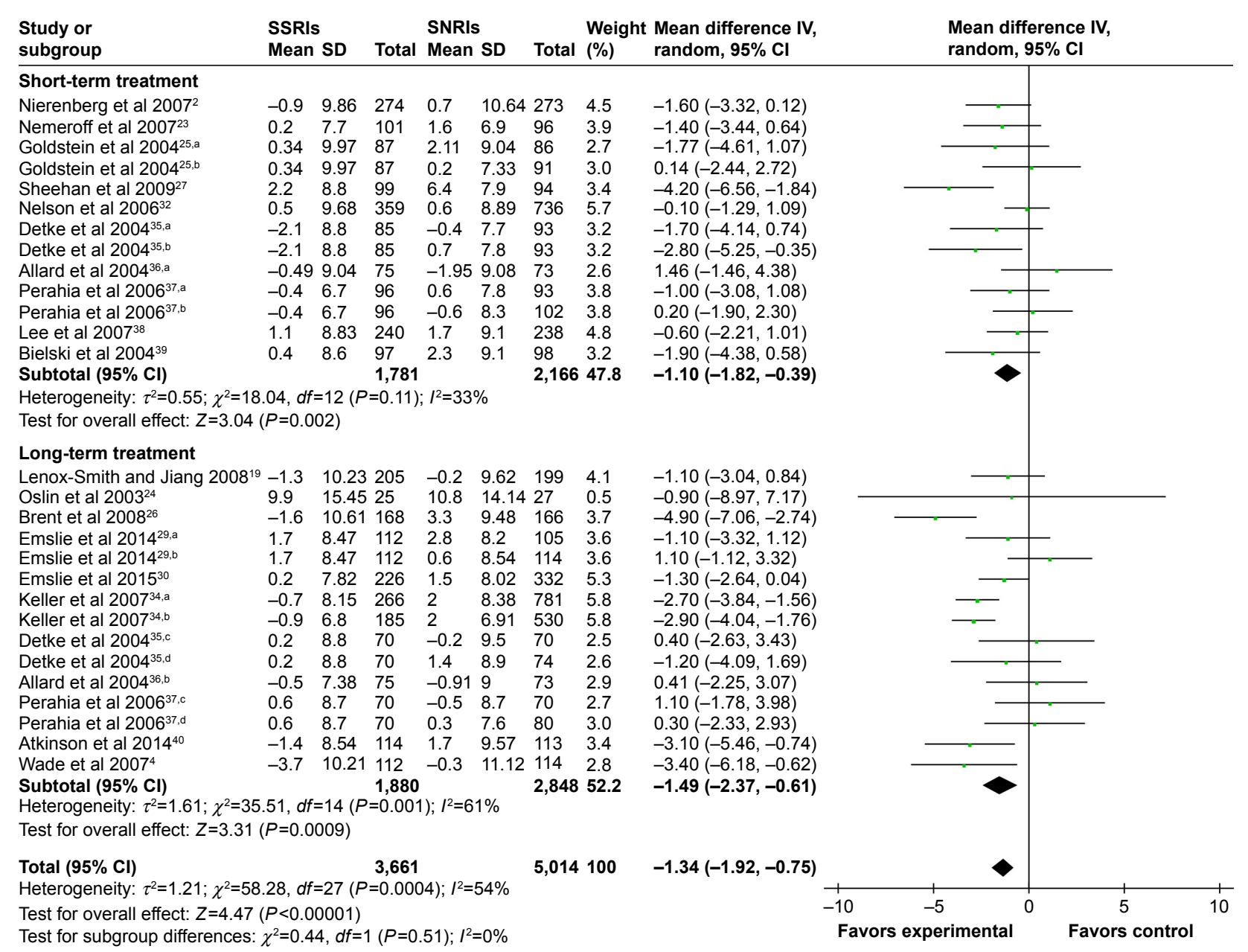

Figure 6 A forest plot of RCTs comparing SSRI group with SNRI group for change in diastolic blood pressure change of short-/long-term duration.

Abbreviations: RCTs, randomized controlled trials; SSRI, selective serotonin reuptake inhibitor; SNRI, serotonin and noradrenaline reuptake inhibitor.

exert effects such as relieving depressive symptoms ${ }^{45}$ yet antidepressant medications could not reliably change BP through depression amelioration. The roles of depression and antidepressant agents affecting BP are regarded complex, and relevant studies are warranted. There exists some evidence suggesting that both SSRIs and SNRIs are related to the autonomic nervous system. SNRIs can exacerbate the situation that autonomic balance shifts to sympathetic predominance, while SSRIs might not affect the balance..$^{11,44}$ Furthermore, sympathetic activity may be attenuated by administration of SSRIs in major depressive patients, ${ }^{46,47}$ thus reducing cardiac risk to some degree. ${ }^{46}$ Sertraline has been demonstrated to decrease sympathetic tone of patients with depression in conditions of physiological stress, ${ }^{47}$ and it may be an appropriate agent for comorbidity of depression and cardiac disease. ${ }^{48}$ SNRIs exhibit a broad spectrum of antidepressant activity, resembling SSRIs, as well as show noradrenergic potentiation. Because of noradrenergic potentiation, the SNRIs can elevate BP to some extent.
Depression deregulates hypothalamic-pituitary-adrenal axis when depressive people are experiencing psychological stress. ${ }^{2}$ As SSRIs and SNRIs improve the stressful condition, we speculate that they can play a role in hypothalamicpituitary-adrenal axis for long-term treatment, which can affect BP to a certain degree.

In addition, this greater increase in BP from baseline in SNRI-treated patients compared with SSRI-treated patients is consistent with the pharmacological actions, which are actions on both serotonergic and noradrenergic neurotransmission and on serotonergic neurotransmission. ${ }^{30} \mathrm{~A} 3 \mathrm{mmHg}$ difference in SBP may be thought not clinically relevant, particularly at older ages. Indeed, even a $2 \mathrm{mmHg}$ lower than usual SBP would involve $\sim 10 \%$ lower stroke mortality and about $7 \%$ lower mortality from ischemic heart disease or other vascular causes in middle age. Therefore, for the general normotensive population, producing persistent reductions in average $\mathrm{BP}$ of just a few $\mathrm{mmHg}$ by some widely practicable methods should avoid large absolute numbers 


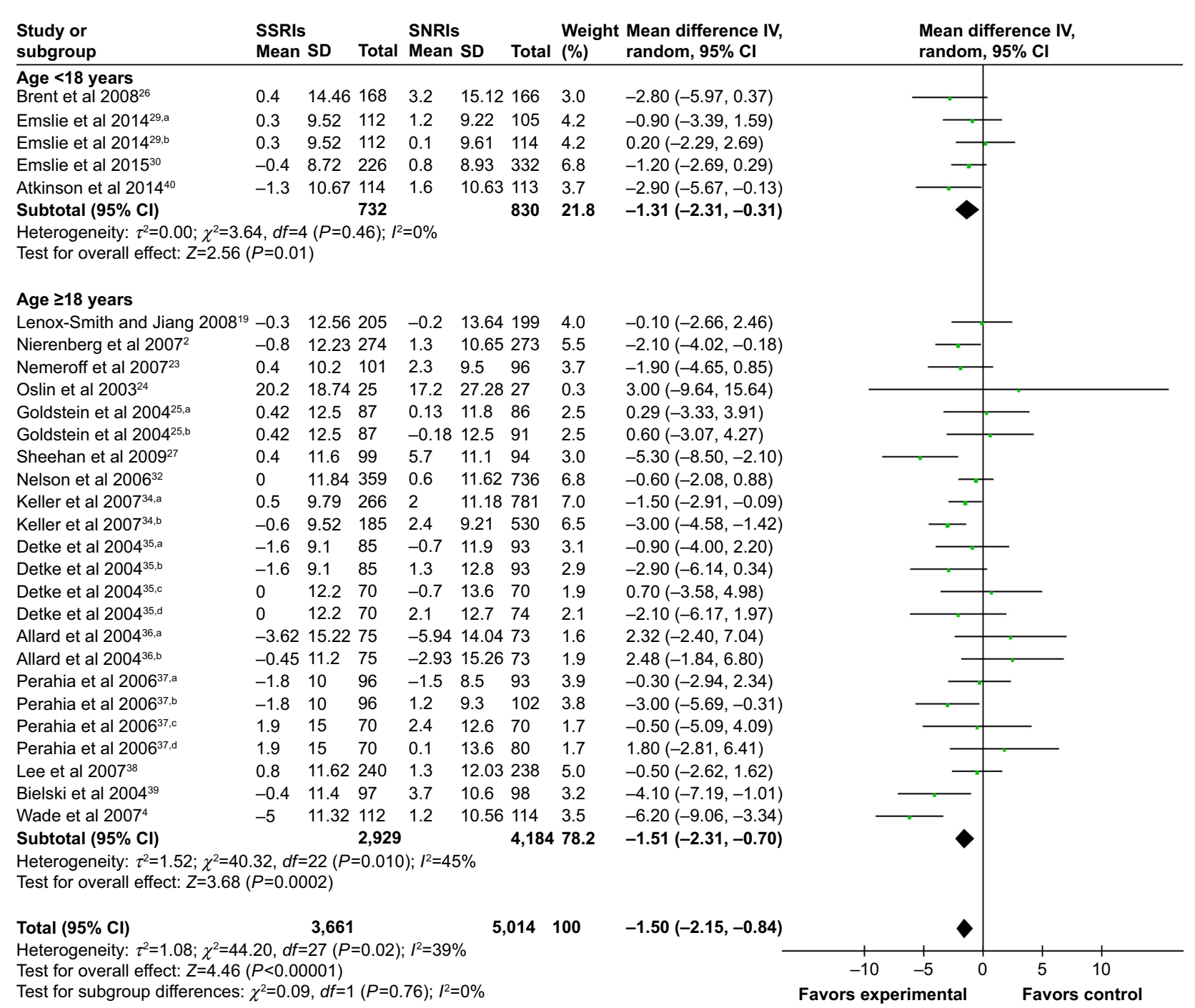

Figure $7 \mathrm{~A}$ forest plot of RCTs comparing SSRI group with SNRI group for change in systolic blood pressure change of different ages.

Abbreviations: RCTs, randomized controlled trials; SSRI, selective serotonin reuptake inhibitor; SNRI, serotonin and noradrenaline reuptake inhibitor.

of premature deaths and disabling strokes. ${ }^{49}$ Depression and coronary heart disease (CHD) are well known to be associated, and in that case patients may suffer from both diseases and be at higher risk for myocardial infarction. ${ }^{42}$ With the existence of the hypothesis that BP can partly explain the association between cardiovascular disease and psychopathology, SSRIs may be more suitable than SNRIs because of improvement in CHD prognosis for depressive patients with CHD. ${ }^{50}$

The included trials recorded office BP, which showed the incapability to reveal the circadian BP rhythm. ${ }^{51}$ There is research indicating that even a mildly depressive mood is associated with larger among-day BP variability (BPV). ${ }^{52}$ Moreover, $\mathrm{BPV}$ is more sensitive in reflecting depressionassociated changes of autonomic function as compared to heart rate variability ${ }^{53}$ Prior studies have shown that a higher morning BP surge is positively correlated with depressive symptoms ${ }^{54}$ and is associated with stroke risk independently in older hypertensive patients. ${ }^{55} \mathrm{Low}$-frequency component of systolic BPV, a surrogate of sympathetic vasomotor tone, can show blunted response with depressive scores, even in the absence of clinically significant alterations in BP, thus acting as a strong predictor of depressive symptoms. In addition, some studies suggest low-frequency component of systolic BPV may be a potential biomarker of neurovascular functioning, contributing to a better understanding of the interaction between MDD and cardiovascular disease ${ }^{56}$ It has been reported that fluvoxamine has a potency to diminish autonomic cardiac activity, and both pharmacological actions and improvement of depressive mood state could change BP level after antidepressant treatment. ${ }^{57}$ However, antidepressant medication studies 


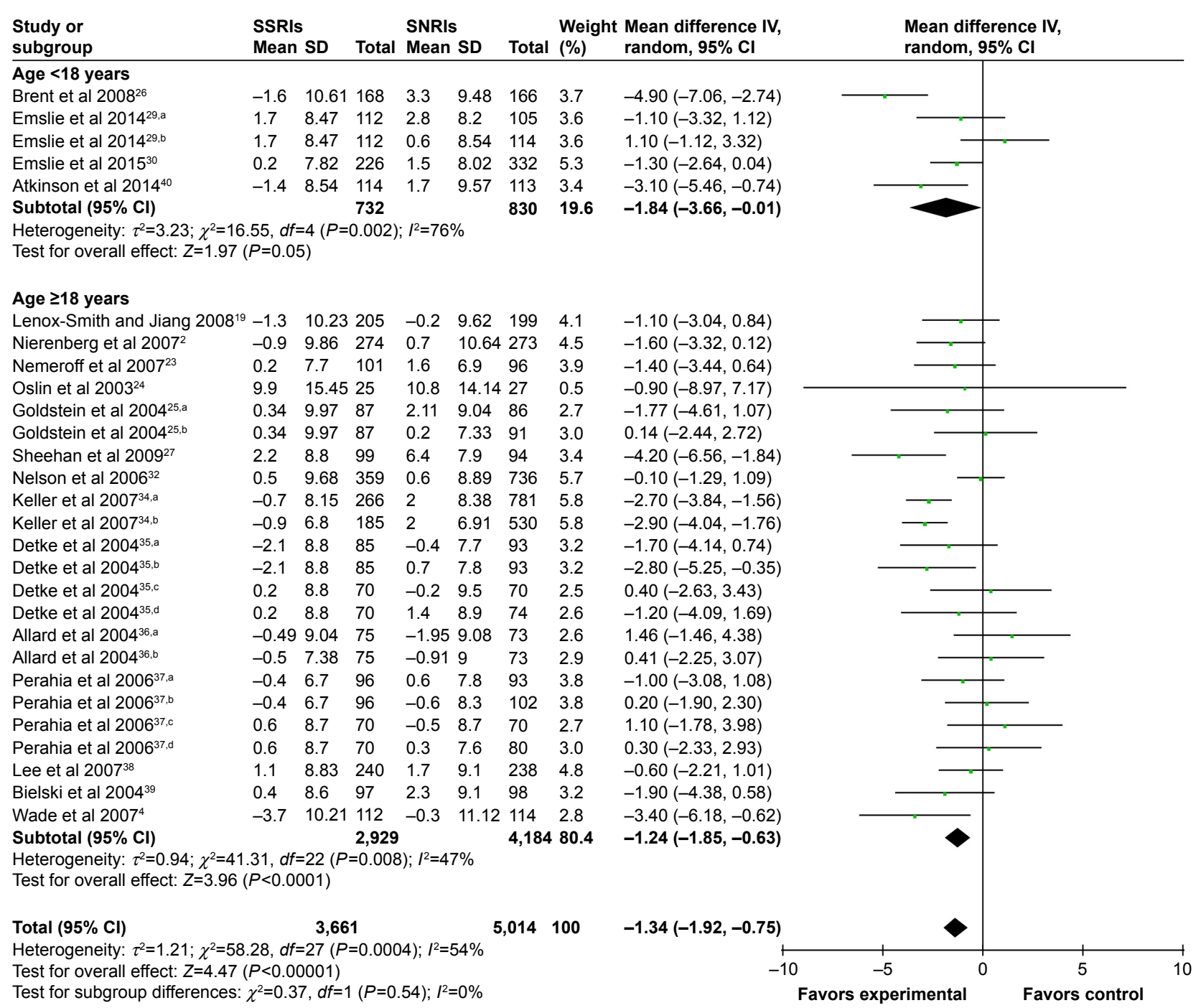

Figure 8 A forest plot of RCTs comparing SSRI group with SNRI group for change in diastolic blood pressure change of different ages.

Abbreviations: RCTs, randomized controlled trials; SSRI, selective serotonin reuptake inhibitor; SNRI, serotonin and noradrenaline reuptake inhibitor.

A

Begg's funnel plot with pseudo

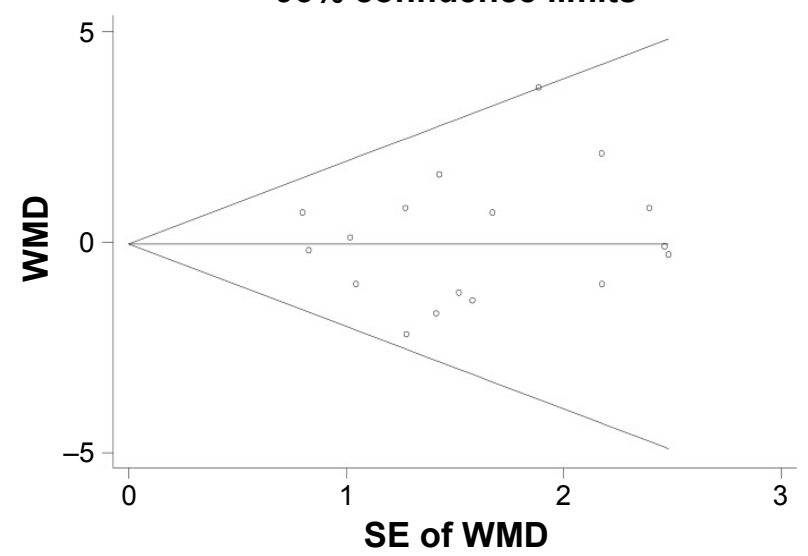

B

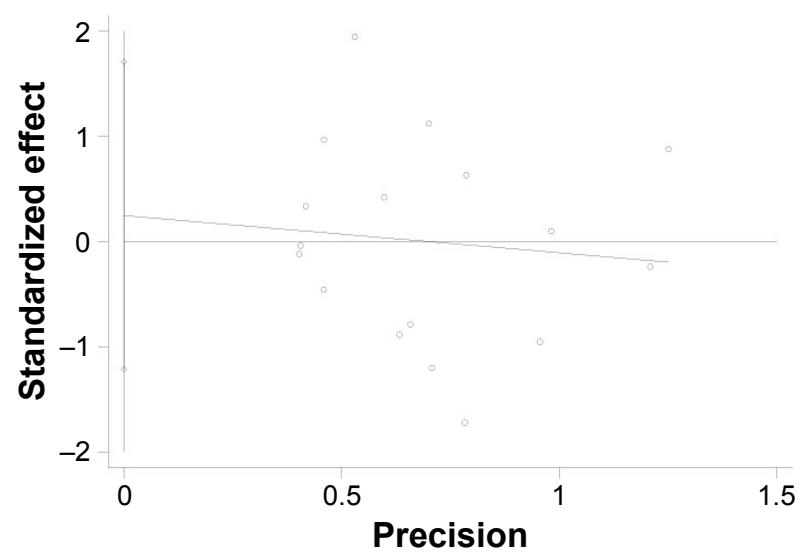

Figure 9 (Continued) 

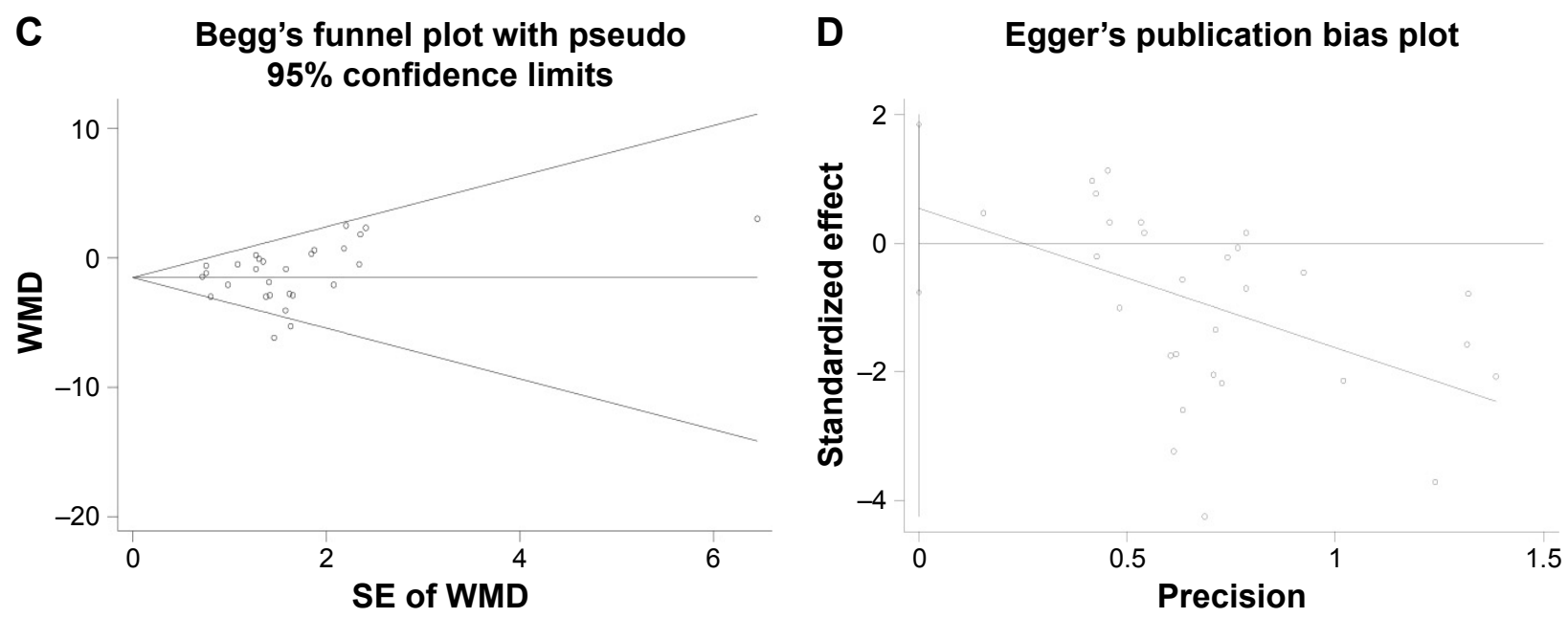

Figure 9 Begg's test and Egger's test identified no publication bias. In the group of SSRI versus placebo: Begg's test: $Z=0.33, P=0.742$ ( $\mathbf{A}$ ); Egger's test: $t=0.36, P=0.724$ (B). In the group of SSRI versus SNRI: Begg's test: $Z=1.19, P=0.236(\mathbf{C})$; Egger's test: $t=0.85, P=0.405$ (D).

Abbreviations: WMD, weighted mean difference; SSRI, selective serotonin reuptake inhibitor; SNRI, serotonin and noradrenaline reuptake inhibitor.

on BPV remain scarce, the effect of antidepressants on autonomic nervous system activity is not clear, and relevant study is warranted.

This meta-analysis, however, had some potential limitations. First, the designs of many RCTs met the inclusion criteria, except when the results did not include details about BP; thus, the exclusion of these RCTs and unpublished data may have resulted in bias. Moreover, six included RCTs reported BP changes for different medication doses or durations, and results were all analyzed as an individual trial according to different doses or durations; therefore, overestimation of the treatment effects on BP might happen. Finally, the trials included adults and children or teenagers, and antihypertensive medications were allowed during treatment, which might lead to bias to some extent, although the doses of cardiovascular medication were fixed.

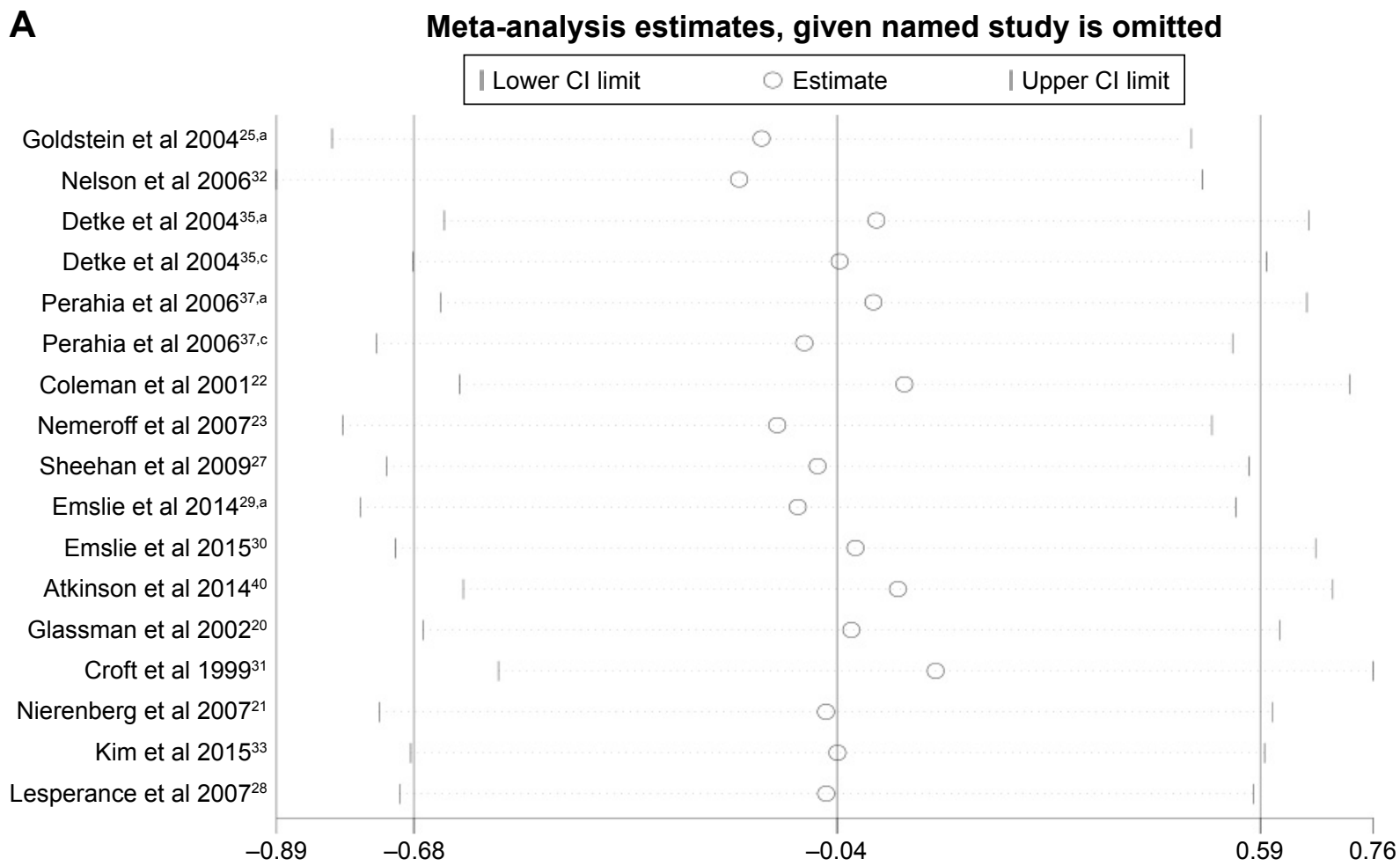

Figure 10 (Continued) 
B

Meta-analysis estimates, given named study is omitted

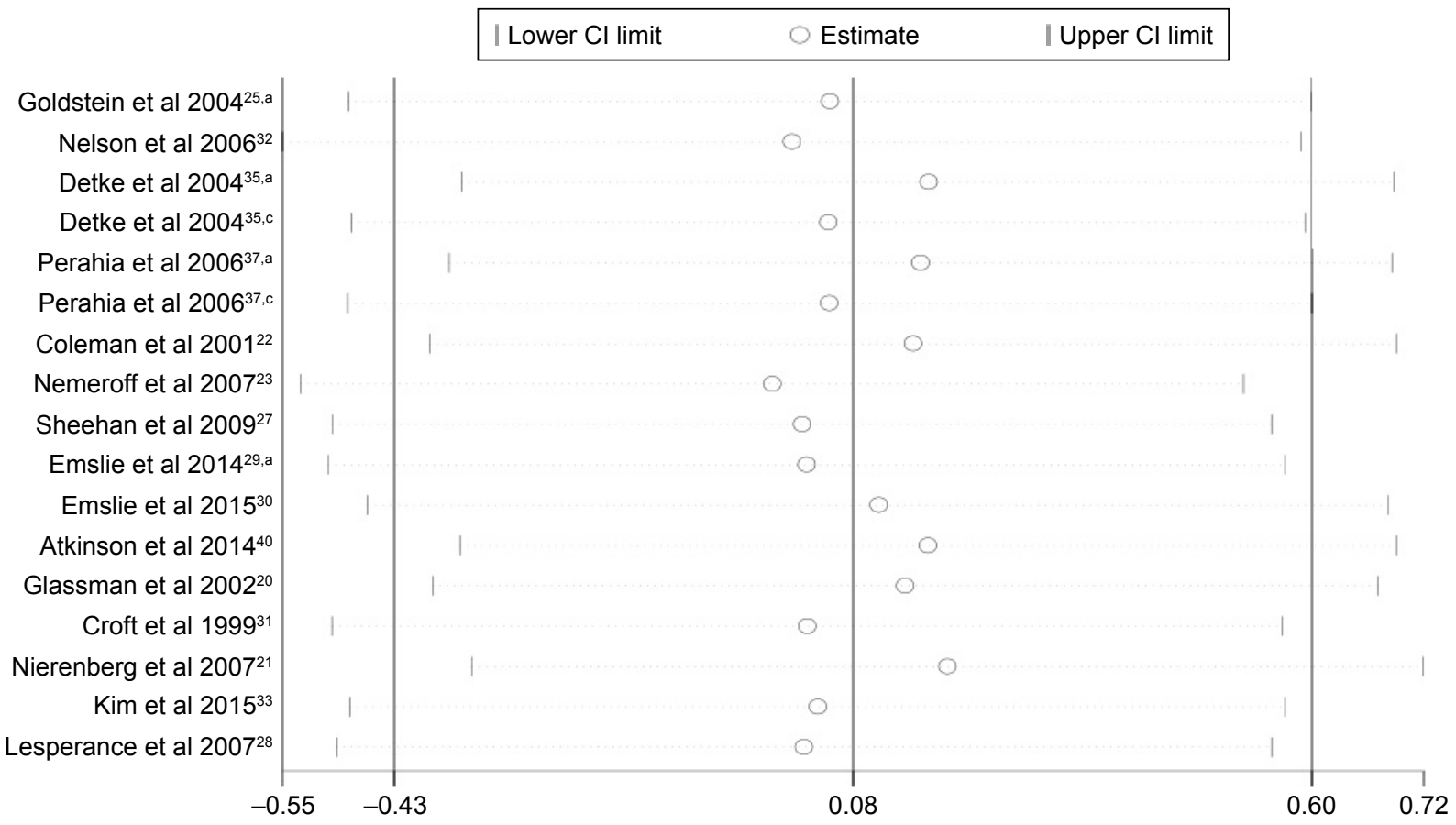

Figure 10 The sensitivity analysis showed the influence of omitting each study in turn in the meta-analysis of SSRI versus placebo. The pooled WMDs (95\% Cl) ranged from $-0.19(-0.89,0.50)$ to $0.10(-0.55,0.76)$ in systolic blood pressure change $(\mathbf{A})$, and from $-0.01(-0.53,0.52)$ to $0.19(-0.34,0.72)$ in diastolic blood pressure change (B), with all showing no statistical significance.

Notes: (A) The sensitivity analysis showed the influence of omitting each study in turn in the meta-analysis of selective serotonin reuptake inhibitor versus placebo in systolic blood pressure change. (B) The sensitivity analysis showed the influence of omitting each study in turn in the meta-analysis of selective serotonin reuptake inhibitor versus placebo in diastolic blood pressure change.

Abbreviations: SSRI, selective serotonin reuptake inhibitor; WMDs, weighted mean differences.

A

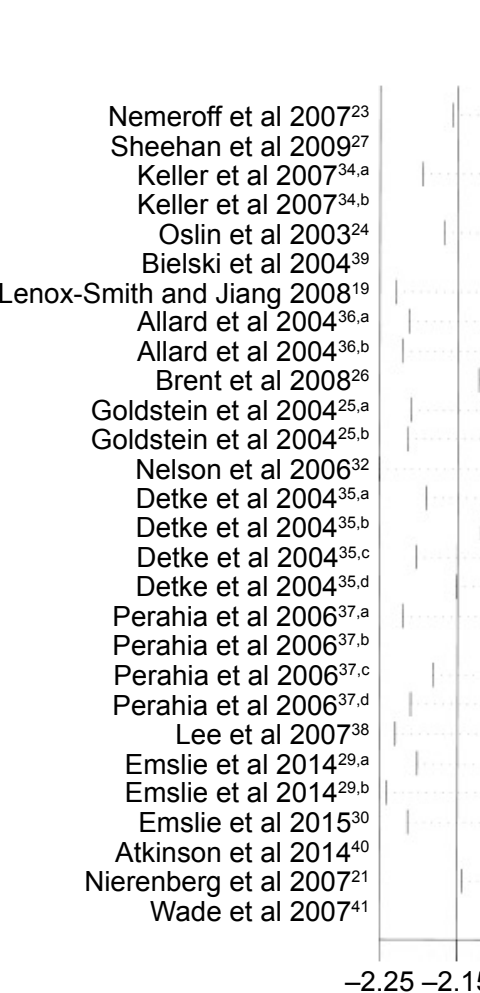

Meta-analysis estimates, given named study is omitted

| Lower Cl limit I Upper Cl limit

Figure I I (Continued) 
B

Meta-analysis estimates, given named study is omitted

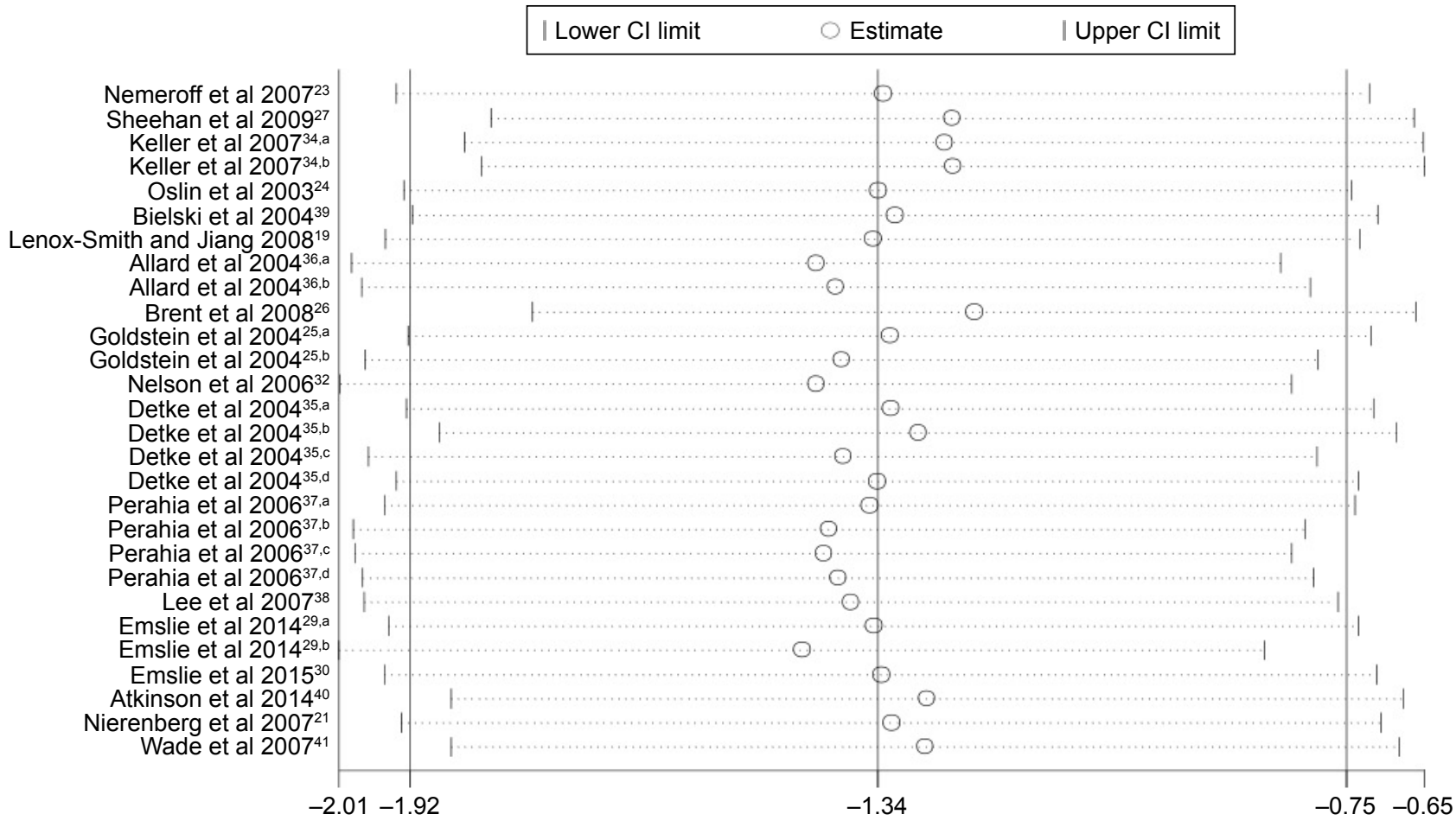

Figure II The sensitivity analysis showed the influence of omitting each study in turn in the meta-analysis of SSRI versus SNRI. The pooled WMDs (95\% CI) ranged from $-1.58(-2.22,-0.93)$ to $-1.36(-1.94,-0.77)$ in systolic blood pressure change $(\mathbf{A})$, and from $-1.43(-2.01,-0.85)$ to $-1.22(-1.77,-0.67)$ in diastolic blood pressure change (B), with all showing statistical significances.

Notes: (A) The sensitivity analysis showed the influence of omitting each study in turn in the meta-analysis of selective serotonin reuptake inhibitor versus serotonin and noradrenaline reuptake inhibitor on systolic blood pressure change. (B) The sensitivity analysis showed the influence of omitting each study in turn in the meta-analysis of selective serotonin reuptake inhibitor versus serotonin and noradrenaline reuptake inhibitor on diastolic blood pressure change.

Abbreviations: SNRI, serotonin and noradrenaline reuptake inhibitor; WMDs, weighted mean differences.

In summary, SSRIs were not established to affect BP fluctuation in depressive patients as a result of their pharmaceutical characteristics or ameliorating depressive symptoms. SNRIs could result in higher SBP and DBP than SSRIs, although none of them caused sudden substantial BP elevation. While office BP is incapable of reflecting the circadian BP rhythm, future research should be focused on antidepressant medication and BPV.

\section{Acknowledgments}

This work was supported by the Guangdong Province Natural Science Foundation (2016A030313319, 2017A030313490) and the Science and Technology Planning Project of Guangdong Province (2014A020212148, 2016B010125001) and Guangzhou city (201510010030).

This work was also supported by Grant [2013]163 from Key Laboratory of Malignant Tumor Molecular Mechanism and Translational Medicine of Guangzhou Bureau of Science and Information Technology and Grant KLB09001 from the Key Laboratory of Malignant Tumor Gene Regulation and Target Therapy of Guangdong Higher Education Institutes.

\section{Disclosure}

The authors report no conflicts of interest in this work.

\section{References}

1. Celano CM, Huffman JC. Depression and cardiac disease: a review. Cardiol Rev. 2011;19(3):130-142.

2. Dhar AK, Barton DA. Depression and the link with cardiovascular disease. Front Psychiatry. 2016;7:33.

3. Scuteri A. Depression and cardiovascular risk: does blood pressure play a role? J Hypertens. 2008;26(9):1738-1739.

4. Hildrum B, Mykletun A, Stordal E, Bjelland I, Dahl AA, Holmen J. Association of low blood pressure with anxiety and depression: the NordTrondelag Health Study. J Epidemiol Community Health. 2007;61(1): 53-58.

5. Hildrum B, Mykletun A, Holmen J, Dahl AA. Effect of anxiety and depression on blood pressure: 11-year longitudinal population study. Br J Psychiatry. 2008;193(2):108-113.

6. Lenoir H, Lacombe JM, Dufouil C, et al. Relationship between blood pressure and depression in the elderly. The Three-City Study. J Hypertens. 2008;26(9):1765-1772.

7. Pan Y, Cai W, Cheng Q, Dong W, An T, Yan J. Association between anxiety and hypertension: a systematic review and meta-analysis of epidemiological studies. Neuropsychiatr Dis Treat. 2015;11: $1121-1130$.

8. Stein DJ, Gureje O. Depression and anxiety in the developing world: is it time to medicalise the suffering? Lancet. 2004;364(9430):233-234.

9. Scalco AZ, Scalco MZ, Azul JB, Lotufo Neto F. Hypertension and depression. Clinics (Sao Paulo). 2005;60(3):241-250. 
10. Licht CM, de Geus EJ, Seldenrijk A, et al. Depression is associated with decreased blood pressure, but antidepressant use increases the risk for hypertension. Hypertension. 2009;53(4):631-638.

11. Thase ME, Larsen KG, Reines E, Kennedy SH. The cardiovascular safety profile of escitalopram. Eur Neuropsychopharmacol. 2013;23(11): 1391-1400.

12. Amsterdam JD, Garcia-Espana F, Fawcett J, et al. Blood pressure changes during short-term fluoxetine treatment. J Clin Psychopharmacol. 1999;19(1):9-14.

13. Feighner JP. Cardiovascular safety in depressed patients: focus on venlafaxine. J Clin Psychiatry. 1995;56(12):574-579.

14. Thase ME. Effects of venlafaxine on blood pressure: a meta-analysis of original data from 3744 depressed patients. J Clin Psychiatry. 1998; 59(10):502-508.

15. Derby MA, Zhang L, Chappell JC, et al. The effects of supratherapeutic doses of duloxetine on blood pressure and pulse rate. J Cardiovasc Pharmacol. 2007;49(6):384-393.

16. Cohen HW, Gibson G, Alderman MH. Excess risk of myocardial infarction in patients treated with antidepressant medications: association with use of tricyclic agents. Am J Med. 2000;108(1):2-8.

17. Nezafati MH, Eshraghi A, Vojdanparast M, Abtahi S, Nezafati P. Selective serotonin reuptake inhibitors and cardiovascular events: a systematic review. J Res Med Sci. 2016;21:66.

18. Maayan L, Vakhrusheva J, Correll CU. Effectiveness of medications used to attenuate antipsychotic-related weight gain and metabolic abnormalities: a systematic review and meta-analysis. Neuropsychopharmacology. 2010;35(7):1520-1530.

19. Lenox-Smith AJ, Jiang Q. Venlafaxine extended release versus citalopram in patients with depression unresponsive to a selective serotonin reuptake inhibitor. Int Clin Psychopharmacol. 2008;23(3): 113-119.

20. Glassman AH, O'Connor CM, Califf RM, et al. Sertraline treatment of major depression in patients with acute MI or unstable angina. JAMA. 2002;288(6):701-709.

21. Nierenberg AA, Greist JH, Mallinckrodt CH, et al. Duloxetine versus escitalopram and placebo in the treatment of patients with major depressive disorder: onset of antidepressant action, a non-inferiority study. Curr Med Res Opin. 2007;23(2):401-416.

22. Coleman CC, King BR, Bolden-Watson C, et al. A placebocontrolled comparison of the effects on sexual functioning of bupropion sustained release and fluoxetine. Clin Ther. 2001;23(7): 1040-1058.

23. Nemeroff CB, Thase ME; EPIC 014 Study Group. A double-blind, placebo-controlled comparison of venlafaxine and fluoxetine treatment in depressed outpatients. J Psychiatr Res. 2007;41(3-4): 351-359.

24. Oslin DW, Ten Have TR, Streim JE, et al. Probing the safety of medications in the frail elderly: evidence from a randomized clinical trial of sertraline and venlafaxine in depressed nursing home residents. J Clin Psychiatry. 2003;64(8):875-882.

25. Goldstein DJ, Lu Y, Detke MJ, Wiltse C, Mallinckrodt C, Demitrack MA Duloxetine in the treatment of depression: a double-blind placebocontrolled comparison with paroxetine. J Clin Psychopharmacol. 2004; 24(4):389-399.

26. Brent D, Emslie G, Clarke G, et al. Switching to another SSRI or to venlafaxine with or without cognitive behavioral therapy for adolescents with SSRI-resistant depression: the TORDIA randomized controlled trial. JAMA. 2008;299(8):901-913.

27. Sheehan DV, Nemeroff CB, Thase ME, Entsuah R; EPIC 016 Study Group. Placebo-controlled inpatient comparison of venlafaxine and fluoxetine for the treatment of major depression with melancholic features. Int Clin Psychopharmacol. 2009;24(2):61-86.

28. Lesperance F, Frasure-Smith N, Koszycki D, et al. Effects of citalopram and interpersonal psychotherapy on depression in patients with coronary artery disease: The Canadian Cardiac Randomized Evaluation of Antidepressant and Psychotherapy Efficacy (CREATE) trial. JAMA. 2007;297(4):367-379.
29. Emslie GJ, Prakash A, Zhang Q, Pangallo BA, Bangs ME, March JS. A double-blind efficacy and safety study of duloxetine fixed doses in children and adolescents with major depressive disorder. J Child Adolesc Psychopharmacol. 2014;24(4):170-179.

30. Emslie GJ, Wells TG, Prakash A, et al. Acute and longer-term safety results from a pooled analysis of duloxetine studies for the treatment of children and adolescents with major depressive disorder. J Child Adolesc Psychopharmacol. 2015;25(4):293-305.

31. Croft H, Settle E Jr, Houser T, Batey SR, Donahue RM, Ascher JA. A placebo-controlled comparison of the antidepressant efficacy and effects on sexual functioning of sustained-release bupropion and sertraline. Clin Ther. 1999;21(4):643-658.

32. Nelson JC, Lu Pritchett Y, Martynov O, Yu JY, Mallinckrodt CH, Detke MJ. The safety and tolerability of duloxetine compared with paroxetine and placebo: a pooled analysis of 4 clinical trials. Prim Care Companion J Clin Psychiatry. 2006;8(4):212-219.

33. Kim JM, Bae KY, Stewart R, et al. Escitalopram treatment for depressive disorder following acute coronary syndrome: a 24-week double-blind, placebo-controlled trial. J Clin Psychiatry. 2015;76(1):62-68.

34. Keller MB, Trivedi MH, Thase ME, et al. The prevention of recurrent episodes of depression with venlafaxine for two years (PREVENT) study: outcomes from the acute and continuation phases. Biol Psychiatry. 2007;62(12):1371-1379.

35. Detke MJ, Wiltse CG, Mallinckrodt CH, McNamara RK, Demitrack MA, Bitter I. Duloxetine in the acute and long-term treatment of major depressive disorder: a placebo- and paroxetine-controlled trial. Eur Neuropsychopharmacol. 2004;14(6):457-470.

36. Allard P, Gram L, Timdahl K, Behnke K, Hanson M, Sogaard J. Efficacy and tolerability of venlafaxine in geriatric outpatients with major depression: a double-blind, randomised 6-month comparative trial with citalopram. Int J Geriatr Psychiatry. 2004;19(12): 1123-1130

37. Perahia DG, Wang F, Mallinckrodt CH, Walker DJ, Detke MJ. Duloxetine in the treatment of major depressive disorder: a placebo- and paroxetine-controlled trial. Eur Psychiatry. 2006;21(6):367-378.

38. Lee $\mathrm{P}, \mathrm{Shu} \mathrm{L}, \mathrm{Xu} X$, et al. Once-daily duloxetine $60 \mathrm{mg}$ in the treatment of major depressive disorder: multicenter, double-blind, randomized, paroxetine-controlled, non-inferiority trial in China, Korea, Taiwan and Brazil. Psychiatry Clin Neurosci. 2007;61(3):295-307.

39. Bielski RJ, Ventura D, Chang CC. A double-blind comparison of escitalopram and venlafaxine extended release in the treatment of major depressive disorder. J Clin Psychiatry. 2004;65(9):1190-1196.

40. Atkinson SD, Prakash A, Zhang Q, et al. A double-blind efficacy and safety study of duloxetine flexible dosing in children and adolescents with major depressive disorder. J Child Adolesc Psychopharmacol. 2014; 24(4):180-189.

41. Wade A, Gembert K, Florea I. A comparative study of the efficacy of acute and continuation treatment with escitalopram versus duloxetine in patients with major depressive disorder. Curr Med Res Opin. 2007; 23(7):1605-1614.

42. Kemp AH, Quintana DS, Gray MA, Felmingham KL, Brown K, Gatt JM. Impact of depression and antidepressant treatment on heart rate variability: a review and meta-analysis. Biol Psychiatry. 2010;67(11): 1067-1074.

43. Rottenberg J. Cardiac vagal control in depression: a critical analysis. Biol Psychol. 2007;74(2):200-211.

44. Koschke M, Boettger MK, Schulz S, et al. Autonomy of autonomic dysfunction in major depression. Psychosom Med. 2009;71(8):852-860.

45. Cipriani A, Furukawa TA, Salanti G, et al. Comparative efficacy and acceptability of 12 new-generation antidepressants: a multipletreatments meta-analysis. Lancet. 2009;373(9665):746-758.

46. Barton DA, Dawood T, Lambert EA, et al. Sympathetic activity in major depressive disorder: identifying those at increased cardiac risk? J Hypertens. 2007;25(10):2117-2124.

47. Scalco AZ, Rondon MU, Trombetta IC, et al. Muscle sympathetic nervous activity in depressed patients before and after treatment with sertraline. J Hypertens. 2009;27(12):2429-2436. 
48. Alvarez W Jr, Pickworth KK. Safety of antidepressant drugs in the patient with cardiac disease: a review of the literature. Pharmacotherapy. 2003;23(6):754-771.

49. Lewington S, Clarke R, Qizilbash N, Peto R, Collins R. Prospective Studies Collaboration. Age-specific relevance of usual blood pressure to vascular mortality: a meta-analysis of individual data for one million adults in 61 prospective studies. Lancet. 2002;360(9349):1903-1913.

50. Pizzi C, Rutjes AW, Costa GM, Fontana F, Mezzetti A, Manzoli L. Meta-analysis of selective serotonin reuptake inhibitors in patients with depression and coronary heart disease. Am J Cardiol. 2011;107(7): 972-979.

51. Symonides B, Holas P, Schram M, Sleszycka J, Bogaczewicz A, Gaciong Z. Does the control of negative emotions influence blood pressure control and its variability? Blood Press. 2014;23(6):323-329.

52. Okajima K, Yamanaka G, Oinuma S, et al. Even mild depression is associated with among-day blood pressure variability, including masked non-dipping assessed by $7-\mathrm{d} / 24-\mathrm{h}$ ambulatory blood pressure monitoring. Clin Exp Hypertens. 2015;37(5):426-432.

53. Voss A, Boettger MK, Schulz S, Gross K, Bar KJ. Gender-dependent impact of major depression on autonomic cardiovascular modulation. Prog Neuropsychopharmacol Biol Psychiatry. 2011;35(4): 1131-1138.
54. FitzGerald L, Ottaviani C, Goldstein IB, Shapiro D. Effects of dipping and psychological traits on morning surge in blood pressure in healthy people. J Hum Hypertens. 2012;26(4):228-235.

55. Kario K, Pickering TG, Umeda Y, et al. Morning surge in blood pressure as a predictor of silent and clinical cerebrovascular disease in elderly hypertensives: a prospective study. Circulation. 2003;107(10): 1401-1406.

56. Sanchez-Gonzalez MA, May RW, Koutnik AP, Kabbaj M, Fincham FD. Sympathetic vasomotor tone is associated with depressive symptoms in young females: a potential link between depression and cardiovascular disease. Am J Hypertens. 2013;26(12):1389-1397.

57. Volkers AC, Tulen JH, van den Broek WW, Bruyn JA, Passchier J, Pepplinkhuizen L. Effects of imipramine, fluvoxamine and depressive mood on autonomic cardiac functioning in major depressive disorder. Pharmacopsychiatry. 2004;37(1):18-25.

\section{Publish your work in this journal}

Neuropsychiatric Disease and Treatment is an international, peerreviewed journal of clinical therapeutics and pharmacology focusing on concise rapid reporting of clinical or pre-clinical studies on a range of neuropsychiatric and neurological disorders. This journal is indexed on PubMed Central, the 'PsycINFO' database and CAS, and is the official journal of The International Neuropsychiatric Association (INA). The manuscript management system is completely online and includes a very quick and fair peer-review system, which is all easy to use. Visit http://www.dovepress.com/testimonials.php to read real quotes from published authors. 